\title{
Tatonnement Beyond Gross Substitutes? Gradient Descent to the Rescue
}

\author{
Yun Kuen Cheung * Richard Cole ${ }^{\dagger} \quad$ Nikhil Devanur ${ }^{\ddagger}$
}

\begin{abstract}
Tatonnement is a simple and natural rule for updating prices in Exchange (ArrowDebreu) markets. In this paper we define a class of markets for which tatonnement is equivalent to gradient descent. This is the class of markets for which there is a convex potential function whose gradient is always equal to the negative of the excess demand. We call this class the Convex Potential Function (CPF) markets. We show the following results.

- CPF markets contain the class of Eisenberg Gale (EG) markets, defined previously by Jain and Vazirani.

- The subclass of CPF markets for which the demand is a differentiable function contains exactly those markets whose demand function has a symmetric negative semi-definite Jacobian.

- We define a family of continuous versions of tatonnement based on gradient descent using a Bregman divergence. As we show, for many CPF markets, every process in this family will converge to an equilibrium and the process based on KL-divergence will converge for even more of these markets. This is analogous to the classic result for markets satisfying the Weak Gross Substitutes property.

- A discrete version of tatonnement converges toward the equilibrium for the following markets of complementary goods; its convergence rate for these settings is analyzed using a common potential function.

- Fisher markets in which all buyers have Leontief utilities; these are all EG markets. (Fisher markets are markets in which all agents are either buyers or sellers.) The tatonnement process reduces the distance to the equilibrium, as measured by the potential function, to an $\epsilon$ fraction of its initial value in $O(1 / \epsilon)$ rounds of price updates, and require $\Omega(1 / \sqrt{\epsilon})$ rounds in the worst case.

- Fisher markets in which all buyers have complementary CES utilities; again, these are all EG markets. Here, the distance to the equilibrium is reduced to an $\epsilon$ fraction of its initial value in $O(\log (1 / \epsilon))$ rounds of price updates.

This shows that tatonnement converges for the entire range of Fisher markets when buyers have complementary CES utilities, in contrast to prior work, which could analyze only the substitutes range, together with a small portion of the complementary range.
\end{abstract}

\footnotetext{
${ }^{*}$ Courant Institute, New York University

${ }^{\dagger}$ Courant Institute, New York University

${ }^{\ddagger}$ Microsoft Research Redmond
} 


\section{Introduction}

General equilibrium theory, the study of markets and their equilibria, has been a core topic in economics for over a century. Informally speaking, an equilibrium is a collection of prices at which the supply and demand of every good in the market balances. Two central questions in general equilibrium theory are whether equilibria exist and if so how to compute them. The issue of existence was settled for a very general setting in 1954 by Arrow and Debreu [2] by means of Katutani's fixed point theorem. However, the computation of equilibria was already being worked on in the 1890s by Fisher, who built a hydraulic apparatus for this task (see [6] for a description). The formal study of this topic began even earlier with the introduction of an equilibrium model by Walras in 1874 [30], along with a natural, simple, distributed price update process which he named tatonnement.

Tatonnement is broadly defined in terms of the following criteria: if the demand for a good is more than the supply, increase the price of the good, and conversely, decrease the price when the demand is less than the supply. The price adjustment for each good is in the direction of its own excess demand and is independent of the demand for other goods. Classically, tatonnement has been thought of as a continuous process, with price adjustments and demand responses happening continuously and instantaneously. A computer science approach is to consider updates at discrete time intervals and to bound the number of updates required (though discrete updates were also considered in the economics literature as early as the 60s [29]).

Implicit in the question of how to compute equilibria is the assumption that markets do reach these equilibria, which raises the further question of how they reach them. Tatonnement is a natural candidate for such a process. An early positive result, due to Arrow, Block and Hurwitz [1], showed that a continuous version of tatonnement converges to an equilibrium for markets satisfying the weak gross substitutes (WGS) property, namely that increasing the price of one good does not decrease the demand for any other good, 1 However, the hope that tatonnement would converge for all markets was dashed by Scarf [27], who showed an example of a market where tatonnement does not converge; in fact, it exhibits cyclic behavior. Thus one can hope to show that tatonnement converges only for specific classes of markets.

The question of how the markets might reach equilibria encompasses many issues. For example, one might ask whether strategic behavior of the market participants is accounted for. This has been considered elsewhere, and it is known that in the limit, as participants or agents become infinitesimal, their strategic power becomes negligible [26]. There have also been some preliminary attempts to quantify this effect in specific markets [9, 8]. One might also ask whether changing supply in addition to prices is an option. This can really be seen as adding production to the model, a thoroughly studied topic in general equilibrium theory. Ultimately, one would like to obtain all-encompassing results, but in this paper we will focus solely on price adjustment, and on tatonnement in particular.

We note that tatonnement is often considered to be an algorithmic process and not a market process; for example, [16] states: "such a model of price adjustment ... describes nobody's actual behavior" (referring to the classic auctioneer explanation of tatonnement). The main point of [16], however, is to give an alternate and more plausible basis for tatonnement. More recently, Cole and Fleischer [12] gave another plausible basis for tatonnement by introducing the Ongoing Market model, in which tatonnement and other price update processes can naturally

\footnotetext{
1 The (strict) gross substitutes property says that increasing the price of one good strictly increases the demand for any other good.
} 
be viewed as in-market processes. The continued interest in the plausibility of tatonnement is also reflected in some experiments by Hirota [19] which show the predictive accuracy of tatonnement in a non-equilibrium trade setting [19].

Returning to the question of convergence, Cole and Fleischer [12] showed that a discrete version of tatonnement converges quite quickly, for a class of markets satisfying the weak gross substitutes property [12]. The current paper is motivated by the quest for other broad classes of markets for which the same holds true. Of particular interest are markets that exhibit complementarity, such as the Constant Elasticity of Substitution (CES) utilities and Leontief utilities. This work will largely focus on Fisher markets, markets in which the participants or agents can be partitioned into buyers and sellers ${ }^{2}$. (See Section 2 for formal definitions.)

For the WGS case, the existing results all rely on very strong properties of WGS markets that are naturally helpful in showing that tatonnement converges. One example of such a property is that for Fisher markets the extreme prices always move in, i.e., the bound on the ratio of the current price to the equilibrium price is guaranteed to shrink [12]. Another example, again for Fisher markets, is that the equilibrium can be reached by starting with very small prices and monotonically increasing them. It is easy to show that such strong properties cease to hold in the complementary regime. Therefore new techniques are needed to handle such markets.

In this paper we relate the tatonnement process to another simple and natural process: gradient descent. Gradient descent is a family of algorithms used to minimize convex functions. It works by starting at some point and moving in the direction of the negative of the gradient. We consider the class of markets for which the tatonnement process is formally equivalent to performing gradient descent on a convex function. In particular, we define the class of Convex Potential Function (CPF) markets to be those markets for which there is a convex potential function whose gradient ${ }^{3}$ is always equal to the negative of the excess demand. We show that this class contains the class of Eisenberg-Gale (EG) markets introduced by Jain and Vazirani [20].

The subclass of CPF markets for which the demand is differentiable can be characterized in terms of the Jacobian! $4^{4}$ of the demand function. These are exactly the markets for which the Jacobian of the demand function is always symmetric and negative semi-definite $5^{5}$ We call this class the Convex Conservative Vector Field (CCVF) markets, since functions that have a symmetric Jacobian are called conservative vector fields. The aforementioned CES and Leontief utilities along with many other interesting markets (in the Fisher market model) are contained in the intersection of EG markets and CCVF markets.

The equivalence with gradient descent opens up the entire tool box developed to analyze gradient descent and provides a principled approach to show convergence of the tatonnement process. For a large class of CPF markets, we show that a continuous version of tatonnement converges to an equilibrium. For the special cases of CES and Leontief utilities, we show stronger con-

\footnotetext{
${ }^{2}$ The term Fisher market was coined by the computer science community to refer to this class of markets, which were the markets defined by Fisher and experimented with in his already mentioned computational work in the $1890 \mathrm{~s}$.

${ }^{3}$ More generally, the potential function need not be differentiable and the demand need not be unique, in which case the equivalence is between the subgradient of the potential function and the set of excess demand vectors.

${ }^{4}$ Recall that the Jacobian of a differentiable function from $\mathbb{R}^{n}$ to $\mathbb{R}^{n}$ is the matrix whose $(i, j)$ entry is the rate of change of the $i^{\text {th }}$ component of the function with respect to a change in the $j^{\text {th }}$ coordinate.

${ }^{5}$ By contrast, if the off-diagonal entries of the Jacobian are all positive, then the market satisfies the weak gross substitutes property.
} 
vergence results by proving certain structural properties of the corresponding convex functions for these markets.

We now summarize the main contributions of the paper.

- The class of Eisenberg-Gale (EG) markets contains all Fisher markets for which the equilibrium allocation is captured by a certain type of convex program called the EisenbergGale-type (EG-type) convex program. We show that EG markets are CPF markets by explicitly constructing a convex potential function (Theorem 4). In fact, the potential function is the objective function of the dual of the corresponding EG-type convex program.

- We show that a family of continuous versions of the tatonnement process converges to the equilibrium for a large class of CPF markets. This family is derived by considering gradient descent with respect to any Bregman divergence and taking the limit as the step size goes to zero (Theorem 22). In addition, the process based on KL-divergence (a particular Bregman divergence) converges for an even larger class of CPF markets. This mirrors the classic result of [1] which shows a similar result for gross substitutes markets.

- For Leontief utilities, we show a a fairly fast rate of convergence for a discrete version of the process, namely, the number of time steps required to reduce the distance from the equilibrium to an $\epsilon$ fraction of its initial value, as measured by the potential function, is $O(1 / \epsilon)$ (Theorem 25) $]^{6}$ This follows from a small modification of a general result of [7] that shows convergence of gradient descent with Bregman divergences at this rate whenever the convex function satisfies a certain sandwiching property 7 We show that the potential function in this case satisfies this sandwiching property for an appropriate choice of parameters with respect to the KL-divergence.

We also show that, in the worst case, tatonnement uses $\Omega(1 / \sqrt{\epsilon})$ iterations with Leontief utilities. Consequently, the linear bounds achieved for CES utilities (see below) cannot extend to Leontief utilities.

- For CES utilities we show a linear convergence, that is, the number of time steps required to reduce the distance from the equilibrium to an $\epsilon$ fraction of its initial value, again as measured by the potential function, is $O(\log (1 / \epsilon))$ (Theorem 35). This is obtained by showing that the potential function in this case satisfies a stronger sandwiching property. This stronger property is reminiscent of strong convexity but to the best of our knowledge, this particular property has not been used before. We also note that when reasonably near to equilibrium, the potential function has value $\Theta\left(\sum_{j} z_{j}^{2} p_{j}\right)$, where $z_{j}$ is the excess demand for good $j$ and $p_{j}$ is its price (Lemmas 32 and 33 ).

In addition, we show that this analysis handles CES utilities that are substitutes, thereby providing an alternate analysis for the results in [12].

\section{Related work}

The stability of the tatonnement process has been considered to be one of the most fundamental issues in general equilibrium theory, and the textbook of Mas-Colell, Whinston and Green [24] contains a good summary of the classic results.

\footnotetext{
${ }^{6}$ The $O()$ hides market dependent parameters.

7 Actually we observe that a slightly weaker version of the property suffices.
} 
More recently, discrete versions of tatonnement have been studied. Codenotti et al. [11] consider a tatonnement-like process that required some coordination among different goods and showed polynomial time convergence for WGS markets. Cole and Fleischer [12] were the first to establish fast convergence for a truly distributed discrete version of tatonnement, once again for a class of WGS markets. Cheung, Cole and Rastogi [10] extended this result slightly beyond WGS markets, to CES utilities for a limited range of parameters ${ }^{8}$ In comparison, our results cover the entire range of parameters for CES utilities. Fleischer et al. [17] also consider price dynamics that are similar to tatonnement but they also need coordination and further, their results concern the average price throughout the process rather than convergence of the sequence.

In a similar spirit to this paper, Birnbaum, Devanur and Xiao [4] considered another distributed process called the Proportional Response (PR) dynamics for the linear utilities case, showed its equivalence to gradient descent with KL-divergence for a different convex function and obtained convergence rates for the process. The PR dynamics works in the space of offers rather than the space of prices, which is why the corresponding convex function is different. For linear utilities, the PR dynamics are more appropriate than tatonnement, especially since the demand function is not continuous. [4] prove a certain convergence result (Theorem 1) which we use in this paper to show convergence for the case of Leontief utilities.

EG markets were defined by Jain and Vazirani [20], after observing that many markets in the Fisher model had similar convex programs that captured the equilibrium. The following is a brief list of such markets: Eisenberg and Gale [15] gave a convex program for the linear utilities case, generalized by Eisenberg [14] to the case of homothetic utilities, Jain et al. [21] for homothetic utilities with production, and Kelly and Vazirani 23] for certain network-flow markets. Jain and Vazirani [20] showed many algorithmic and structural properties of such markets.

\section{Preliminaries}

A Walrasian market model has $m$ divisible goods and $n$ agents. Each agent $i$ has a utility function $u_{i}: \mathbb{R}_{+}^{m} \rightarrow \mathbb{R}$ that specifies the agent's utility for a given bundle of goods. Each agent $i$ has an initial endowment of $e_{i j}$ amount of good $j$. The supply of good $j, w_{j}:=\sum_{i} e_{i j}$ is the total endowment of good $j$ among all the agents. W.l.o.g. we choose the units of measurement such that the supplies are all 1 . Suppose we assign a price $p_{j}$ to each good $j$, then the demand of agent $i$ is a bundle of goods $\left(x_{i 1}, x_{i 2}, \ldots, x_{i m}\right)$ that maximizes her utility subject to the budget constraint, namely that she does not spend more than the value of her endowment. It is the solution to the following optimization problem:

$$
\begin{aligned}
& \operatorname{maximize} u_{i}\left(x_{i 1}, x_{i 2}, \ldots, x_{i m}\right) \\
& \text { s.t. } \sum_{j} p_{j} x_{i j} \leq \sum_{j} p_{j} e_{i j}, \\
& \quad \forall j, x_{i j} \geq 0 .
\end{aligned}
$$

If the utility function is strictly concave, then there is a unique utility maximizing bundle when the prices are all positive, so we can talk of the demand of an agent. The market demand for

\footnotetext{
${ }^{8} \mathrm{CES}$ utilities are parameterized by an exponent, $\rho$. When $0<\rho \leq 1$ the market is WGS, and when $\rho<0$ the goods are complementary. [10] analyzed the range $-1<\rho \leq 0$.
} 
a good $j$ is $x_{j}=\sum_{i} x_{i j}$, the total demand for that good. This is viewed as a function of the price vector $p=\left(p_{1}, p_{2}, \ldots, p_{m}\right)$. A price $p$ is an equilibrium price if the market clears, that is

$$
\text { for all } j, \quad x_{j}=w_{j}=1 .
$$

For notational convenience, we define the excess demand for good $j$ as $z_{j}=x_{j}-1$. The equilibrium condition is that every excess demand be zero. It is known that equilibrium prices exist if the utility functions are all strictly concave.

An alternate mode is the Fisher market model, where there is a fixed endogenous supply of each good (which is again chosen to be 1 unit). The agents have a fixed endowment of money, which defines their budget constraint. Let the endowment of agent $i$ be $e_{i}$ units of money. The budget constraint for agent $i$ is $\sum_{j} p_{j} x_{i j} \leq e_{i}$. The Fisher model is actually a special case of the exchange model.

We now define some interesting subclasses of markets. A market satisfies the Weak Gross Substitutes (WGS) property or equivalently a market is a WGS market if increasing the price of any one good does not reduce the demand for any other good. If the demand function is continuous and differentiable, then this property can be written as

$$
\frac{\partial x_{j}}{\partial p_{j^{\prime}}} \geq 0, \quad \forall j \neq j^{\prime}
$$

In terms of the Jacobian of the demand function, in a WGS market all the off-diagonal entries are non-negative.

The Leontief utilities are of the form $u_{i}=\min _{j}\left\{x_{i j} / b_{i j}\right\}$. One needs $b_{i j}$ units of good $j$, for each good, in order to get one unit of utility. Thus Leontief utilities capture the case of perfect complements. It is easy to see that the demand for good $j$ is

$$
x_{i j}=\beta_{i} b_{i j}, \quad \text { where } \beta_{i}=e_{i} / \sum_{j} b_{i j} p_{j}
$$

Thus the maximum utility buyer $i$ can obtain is

$$
u_{i}=e_{i} / \sum_{j} b_{i j} p_{j}
$$

Utilities with a Constant Elasticity of Substitution (CES) utilities, are of the form

$$
u_{i}=\left(a_{i 1} x_{1}^{\rho_{i}}+a_{i 2} x_{2}^{\rho_{i}}+\cdots+a_{i m} x_{m}^{\rho_{i}}\right)^{1 / \rho_{i}}
$$

with $\rho_{i} \leq 1$ and $a_{i j} \geq 0$. If $0<\rho_{i} \leq 1$ then the goods are substitutes; the goods are complementary when $\rho_{i}<0$. Leontief utilities are obtained in the limit, as $\rho \rightarrow-\infty$. The utility function obtained in the limit, as $\rho \rightarrow 0$, is called the Cobb-Douglas utility.

An Eisenberg-Gale-type convex program is a convex program of the form

$$
\begin{array}{ll}
\operatorname{maximize} & \sum_{i} e_{i} \log u_{i}\left(x_{i 1}, x_{i 2}, \ldots, x_{i m}\right) \\
\text { s.t. } & \forall j, \quad \sum_{i} x_{i j} \leq 1, \quad(\text { supply constraints) } \\
\forall i, j, \quad x_{i j} \geq 0 .
\end{array}
$$


The base of the log does not matter for the maximization in the convex program. However, later in the paper some calculations are simplified if we assume that the natural logarithm is intended, and so we assume this henceforth.

We note that the above program satisfies Slater's conditions for strong duality (see [5], p. 226, for example) and consequently an optimal solution to the dual problem yields the same optimizing value as the primal program.

An Eisenberg-Gale (EG) market is a Fisher market for which the optimal solution and the (corresponding) Lagrange multipliers of the supply constraints in the above convex program are respectively equilibrium demands and prices for the market. Conversely, equilibrium demands and prices are respectively an optimal solution and Lagrange multipliers of the supply constraints to the above convex program. Note that any strictly monotone transformation of the utility function leaves the market unchanged, since the demand function is invariant under such transformations. Thus one may need to apply suitable monotone transformations to the utility functions in order to obtain an EG market. It is known that buyers with Leontief and CES utilities in the Fisher model form EG markets.

We next present a generalized version of gradient descent and a convergence result for this version. For any strictly convex differentiable function $h$, the Bregman divergence with kernel $h$ is defined as

$$
d_{h}(p, q)=h(p)-h(q)-\nabla h(q) \cdot(p-q) .
$$

For example, the square of the Euclidean distance is obtained as a Bregman divergence, $\| p-$ $q \|^{2}=d_{h}(p, q)$, if $h(p)=\frac{1}{2}\|p\|^{2}$. Another well-known example is the KL-divergence, $\sum_{j} p_{j} \log p_{j}$, which is obtained when

$$
h(p)=\sum_{j} p_{j} \log p_{j}-p_{j}
$$

For a convex function $\phi$, define the tangent hyperplane at a given point $q$, thought of as a linear approximation to the function, as

$$
\ell_{\phi}(p ; q)=\phi(q)+\nabla \phi(q) \cdot(p-q)
$$

where $\nabla \phi(q)$ denotes an arbitrary subgradient of $\phi$ at $q$. The generalized gradient descent w.r.t. a Bregman divergence $d_{h}$ on the convex function $\phi$ is a sequence $p^{0}, p^{1}, \ldots, p^{t} \ldots$, defined inductively (for any given starting point $p^{0}$ ) by

$$
p^{t+1}=\arg \min _{p}\left\{\ell_{\phi}\left(p ; p^{t}\right)+d_{h}\left(p, p^{t}\right)\right\} .
$$

Note that if the subgradient is not unique, then this sequence need not be unique either.

For the quadratic kernel, $h(p)=\frac{1}{2}\|p\|^{2}$, the above update rule reduces to the usual gradient descent rule:

$$
p^{t+1}=p^{t}-\nabla \phi\left(p^{t}\right) .
$$

If the kernel is the weighted entropy, $h(p)=\sum_{j} \gamma_{j}\left(p_{j} \log p_{j}-p_{j}\right)$ for some weights $\gamma_{j}$, the update rule is

$$
p_{j}^{t+1}=p_{j}^{t} \exp \left(\frac{-\nabla_{j} \phi\left(p^{t}\right)}{\gamma_{j}}\right), \quad \text { for all } j .
$$

Birnbaum, Devanur and Xiao [4] showed the following convergence result for gradient descent (6). 
Theorem 1 ([4]). Suppose that the convex function $\phi$ and the kernel $h$ satisfy: for all $p, q$,

$$
\phi(p) \leq \ell_{\phi}(p ; q)+d_{h}(p, q) .
$$

Let $p^{*}$ be a minimizer of $\phi$. Then for all $t$,

$$
\phi\left(p^{t}\right)-\phi\left(p^{*}\right) \leq \frac{d_{h}\left(p^{*}, p^{0}\right)}{t} .
$$

We need a slightly more general version of this theorem where we require (8) to hold only for consecutive pairs $p^{t}, p^{t+1}$ for all $t$, instead of requiring it for all pairs $p, q$. It is easy to see that their proof needs only this weaker condition, yielding the following theorem.

Theorem 2. Suppose that the sequence of prices $p^{t}$ obey the following condition:

$$
\phi\left(p^{t+1}\right) \leq \ell_{\phi}\left(p^{t} ; p^{t+1}\right)+d_{h}\left(p^{t}, p^{t+1}\right) .
$$

Let $p^{*}$ be a minimizer of $\phi$. Then for all $t$,

$$
\phi\left(p^{t}\right)-\phi\left(p^{*}\right) \leq \frac{d_{h}\left(p^{*}, p^{0}\right)}{t} .
$$

The discrete version of the tatonnement process we consider will be equivalent to the gradient descent (6) where $h$ is the weighted entropy function, i.e., the update (7) for a suitable choice of weights $\gamma_{j}$. The potential function $\phi$ will satisfy $\nabla_{j} \phi=-z_{j}$. The continuous versions we consider are obtained by introducing a multiplier $1 / \epsilon$ to the divergence term $d_{h}$ and taking the limit as $\epsilon \rightarrow 0$. This will be presented in more detail in Section 4 .

\subsection{New Definitions}

We now define the new classes of markets being introduced in this paper. A market is said to be a Convex Potential Function (CPF) market if there is a convex potential function $\phi$ of the prices such that for all prices $p, \nabla \phi(p)=-z(p)$. By abuse of notation, we let $\nabla \phi$ denote the set of sub-gradients when $\phi$ is not differentiable $9^{9}$ and we let $z(p)$ denote the set of excess demand vectors when the demand is not unique. The subclass of CPF markets for which the demand function is differentiable is called the Convex Conservative Vector Field (CCVF) markets. The following characterization of CCVF markets follows essentially immediately from Green's Theorem [18, 25].

Lemma 3. A market with a differentiable demand function is CCVF if and only if the Jacobian of its demand function is always a negative semi-definite symmetric matrix.

Proof. For a CCVF market, the potential function satisfies $\nabla \phi(p)=-z(p)$. As $x(p)$ and hence $z(p)$ are differentiable, it is now easy to check that the Jacobian is symmetric. Negative semi-definiteness follows because the potential function $\phi$ associated with the CCVF market is convex, and hence the Jacobian of $-z(p)$ is positive semi-definite.

If the Jacobian of $x(p)$ is symmetric, by Green's Theorem [18, 25], there is a function $f: \mathbb{R}^{n} \rightarrow \mathbb{R}$ such that $\nabla f=x$. Let $\phi=\sum_{j} p_{j}-f(p)$. Then $\nabla \phi(p)=\mathbb{1}-x(p)=-z(p)$. $\phi(p)$ is convex as its Jacobian is positive semi-definite, and as $\nabla \phi(p)=-z(p)$, it follows that the market is a $\mathrm{CPF}$ market with a differentiable demand, i.e. it is a CCVF market.

Markets with Leontief utilities and those with CES utilities are both CCVF markets. By contrast, markets with linear additive utilities are not CCVF.

\footnotetext{
${ }^{9} \mathrm{We}$ assume throughout that $\phi$ is continuous.
} 


\section{EG markets}

In this section we prove the following theorem.

Theorem 4. All EG markets are CPF markets.

The proof is by an explicit construction of a convex potential function $\phi$ for which $\nabla \phi(p)=$ $-z(p) . \phi$ is actually the dual of the corresponding EG-type convex program. Recall that the EG-type convex program has variables $x_{i j}$ for all $i$ and $j$. We let $X$ denote the set of all these variables. Also recall that the optimum solution gives the equilibrium allocation and the optimal Lagrangian multipliers of the supply constraints in the program are the equilibrium prices. The KKT conditions characterize the optimal solution to a convex program and the corresponding Lagrange multipliers. We now write the KKT conditions in terms of the Lagrangian function, which is obtained by multiplying the supply constraints by the prices and adding them to the objective function.

$$
L(X, p):=\sum_{i} e_{i} \log \left(u_{i}\right)-\sum_{i, j} p_{j} x_{i j}+p \cdot \mathbb{1}
$$

on the domain $\left\{X, p: \forall i, j, x_{i j} \geq 0 ; \forall j, p_{j} \geq 0\right\} . X^{*}$ and $p^{*}$ are said to satisfy the KKT conditions if

$$
\begin{aligned}
& \text { 1. } X^{*} \in \arg \max _{X \geq 0} L\left(X, p^{*}\right) \text { and } \\
& \text { 2. } p^{*} \in \arg \min _{p \geq 0} L\left(X^{*}, p\right) \text {, which is equivalent to } \\
& \quad \text { for all } j, \quad p_{j}^{*} \cdot\left(1-\sum_{i} x_{i j}^{*}\right)=0 \text {. }
\end{aligned}
$$

We define the potential function to be the dual objective of the EG-type convex program.

$$
\phi(p):=\max _{X \geq 0} L(X, p) .
$$

$\phi$ is convex by construction. Theorem 4 follows by showing that the gradient of $\phi$ is equal to the negative of the excess demand (Lemma 6). However, the key property of EG markets is captured by the following lemma.

Lemma 5. For an EG market, for all $p$, the demand set $x(p)$ is exactly equal to $\arg \max _{X \geq 0} L(X, p)$, whenever they are both finite.

Proof. Part 1, $x(p) \subseteq \arg \max _{X \geq 0} L(X, p)$ : We first argue that if $x(p)$ is the demand at price $p$ then it must also maximize $L(X, p)$. In fact, we first argue it for the special case when the price and the demand form an equilibrium, denoted by $p^{*}$ and $x\left(p^{*}\right)$. Since this is an EG market, by definition of an EG market, the pair $\left(p^{*}, x\left(p^{*}\right)\right)$ must correspond to an optimal solution of the corresponding convex program. They must therefore satisfy the corresponding KKT conditions (10), which imply that $x\left(p^{*}\right) \in \arg \max _{X \geq 0} L\left(X, p^{*}\right)$ as desired. This immediately shows the same for any price $p$ and every demand $x(p)$, since the pair forms an equilibrium when the supply is equal to $x(p)$. Thus the above holds for all prices and for all demand vectors.

Part 2, $\arg \max _{X \geq 0} L(X, p) \subseteq x(p)$ : The argument is similar to Part 1. Consider any $p$ and an $X$ that maximize $L(X, p)$. Consider the market instance with supply equal to $\sum_{i} x_{i j}$ for 
good $j$. Note that the KKT conditions (10) are then satisfied with $p$ and $X$ for this instance and therefore they form an optimal solution to the corresponding EG-type convex program. Since any optimal solution to the convex program must also be an equilibrium, it follows that $X$ must be a demand at price $p$ as desired.

In fact it is easy to see that the converse of Lemma 5 is also true, that if for all $p$ the demand set is equal to $\arg \max _{X \geq 0} L(X, p)$ then the market is an EG market. The KKT conditions (10) are then exactly the same as the equilibrium conditions.

Lemma 6. $\nabla \phi(p)=\mathbb{1}-x(p)=-z(p)$.

Proof. It is well known that if a convex function is defined as the maximum of many linear functions then the gradient is given by the gradient of the linear function providing this maximum. $\phi$ is indeed defined in this way and by Lemma 5 the arg max'es are given by the demands, or in other words the maximizing linear function $L(X, p)$ is the one defined using the demands. Thus $\nabla \phi(p)=\mathbb{1}-x(p)=-z(p)$.

The following convenient form for $\phi(p)$ was shown in [13], and will be used in the analyses of the markets with Leontief and CES utilities.

Lemma 7. For EG markets with linear, CES or Leontief utilities (and others) the dual objective can be written as

$$
\phi(p)=\sum_{j} p_{j}-\sum_{i} e_{i} \log \left(\nu_{i}\right)+\text { a constant independent of } p
$$

where $\nu_{i}$ is the ratio of $e_{i}$ to the optimal utility of $i$ at price $p$, i.e. the minimum cost for obtaining one unit of utility.

Proof. Recall that

$$
\phi(p):=\max _{X \geq 0} L(X, p)=\max _{X \geq 0}\left\{\sum_{j} p_{j}+\sum_{i} e_{i} \log u_{i}\left(x_{i}\right)-\sum_{i, j} p_{j} x_{i j}\right\},
$$

where $x_{i}$ denotes the demands of buyer $i$. From Lemma5, for each $i$, an $x_{i j}$ in the $\arg \max$ above is buyer $i$ 's demand for good $j$ and therefore $\sum_{j} p_{j} x_{i j}$ must be equal to $e_{i}$. Hence $\sum_{i, j} p_{j} x_{i j}=$ $\sum_{i} e_{i}$ is a constant. We also rewrite $\sum_{i} e_{i} \log u_{i}\left(x_{i}\right)=\sum_{i}-e_{i} \log \left[e_{i} / u_{i}\left(x_{i}\right)\right]+\sum_{i} e_{i} \log e_{i}$; then setting $\nu_{i}=e_{i} / u_{i}\left(x_{i}\right)$ gives $\phi$ in the desired form.

\section{Convergence of Continuous Time Tatonnement}

A continuous version of tatonnement is a trajectory in the price space which, to be notationally consistent with the discrete version, is denoted by $p^{t}$ for all $t \in \mathbb{R}_{+}$. Classically, the trajectory is defined by specifying a differential equation $\frac{d p}{d t}=F(t, p(t))$ for all $t$, which we also call the "update rule". We define a family of update rules derived from gradient descent. As before, 
let $h$ be a strictly convex differentiable function. The natural way to specify the differential equation is

$$
\begin{aligned}
p(\epsilon) & :=\arg \min _{p}\left\{\nabla \phi\left(p^{t}\right) \cdot\left(p-p^{t}\right)+\frac{1}{\epsilon} d_{h}\left(p ; p^{t}\right)\right\} . \\
\frac{d p_{j}}{d t} & :=\lim _{\epsilon \rightarrow 0} \frac{p_{j}(\epsilon)-p_{j}^{t}}{\epsilon} .
\end{aligned}
$$

However, there are three issues we need to address with this specification. The first issue is that in the markets we consider, the demand function of an agent can be multi-valued at a price vector ${ }^{10}$ and hence $\nabla \phi\left(p^{t}\right)$ can also be a set of multiple elements, namely the set of subgradients of $\phi$ at $p^{t}$. Since $\nabla \phi\left(p^{t}\right)$ can be multi-valued, $p(\epsilon)$ and hence $\frac{d p_{j}}{d t}$ can be too. To resolve this, we need the notion of differential inclusion, which is a generalization of differential equations. In brief, a differential inclusion is a system which allows $\frac{d p}{d t}$ to take any value from a set. We specify our class of differential inclusions in the domain $\mathbb{R}_{+}^{n}$, as follows:

$$
\begin{aligned}
p^{t}(\vec{v}, \epsilon) & :=\arg \min _{p}\left\{\vec{v} \cdot\left(p-p^{t}\right)+\frac{1}{\epsilon} d_{h}\left(p ; p^{t}\right)\right\} \\
F\left(p^{t}\right) & :=\left\{\lim _{\epsilon \rightarrow 0} \frac{p^{t}(\vec{v}, \epsilon)-p^{t}}{\epsilon} \mid \vec{v} \in \nabla \phi\left(p^{t}\right)\right\} \\
\frac{d p}{d t} & : \in F\left(p^{t}\right) .
\end{aligned}
$$

The existence of a solution to 13 requires $F$ to be non-empty, convex, compact and upper semi-continuous. (We will give precise definitions and state the relevant results in Section 4.1; we refer the readers to Smirnov's text [28] for more detail on this topic.) In fact, going to set-valued maps also helps us handle some discontinuities, since upper semi-continuity for set-valued maps is in a sense a weaker requirement than continuity for functions.

The second issue is related to the requirement that $F$ be non-empty and compact. Lemma 11 shows that if $\nabla \phi$ is finite and bounded, then $\lim _{\epsilon \rightarrow 0} \frac{p(\vec{v}, \epsilon)-p}{\epsilon}$ exists and is bounded. The main difficulty in showing $\nabla \phi$ is bounded occurs when one of the prices tends to zero. This is also related to the next issue. Prices tending to $\infty$ create a similar difficulty.

The third issue is that we do not allow prices to be negative. This imposes a boundary on the price domain. Classically, existence theorems for differential inclusions/equations guarantee the existence of a solution up to the boundary, i.e. a solution may only be guaranteed for a finite time span. Yet we want global existence, i.e. a solution for $t \in[0,+\infty)$. To help resolve this, we will extend $F(p)$ to the negative price domain so as to remove the boundary while ensuring that any solution remains in $\mathbb{R}_{+}^{n}$.

As we mentioned, the second and the third issues are connected to the main impediment to proving the convergence of tatonnement, which is the possible presence of zero-valued prices on the tatonnement path. If we are using a rule such as the multiplicative update rule, $\frac{d p_{j}^{t}}{d t}=z_{j}^{t} p_{j}^{t}$, the price $p_{j}$ will not change if equal to zero, precluding convergence to an equilibrium with $p_{j}^{*} \neq 0$. One way to avoid this difficulty is to limit the update rule so as to ensure that if a price starts out positive, it will remain positive. Note that this does not preclude a price converging to zero as $t \rightarrow \infty$, if that is its value at equilibrium. In this case, the price domain boundary is never reached.

\footnotetext{
${ }^{10}$ An example: if a buyer has utility function $u\left(x_{1}, x_{2}\right)=x_{1}+3 x_{2}$ and budget 40 , then at prices $\left(p_{1}, p_{2}\right)=$ $(2,6)$, the buyer optimizes her utility by purchasing $\left(x_{1}, x_{2}\right)=(20-3 y, y)$, for any $y \in[0,20 / 3]$.
} 
By contrast, if the price update rule is additive, e.g. $\frac{d p_{j}^{t}}{d t}=z_{j}^{t}$, a price might take on a zero value despite being positive initially. However, this will still be viable so long as the tatonnement avoids price vectors $p$ with $p_{j}=0$ and $z_{j}=\{\infty\}$, which we call unbounded demand price vectors. As we shall see, for linear, CES and Leontief Fisher markets, if they start from a nonunbounded (i.e. from a bounded) demand price vector, they will reach only bounded demand price vectors, regardless of which tatonnement rule is used. In this case, we do need to ensure that the price domain boundary is not crossed.

A solution to the differential inclusion 13$)$ could seek to leave the domain $\mathbb{R}_{+}^{n}$ if $F_{j}\left(p^{t}\right)$ contains a negative value when $p_{j}^{t}=0$, as it may. ${ }^{11}$ But, when $p_{j}^{t}=0, F_{j}\left(p^{t}\right)$ can always be made to contain items with $z_{j} \geq 0$, providing the hope of a solution that remains in $\mathbb{R}_{+}^{n}$. To this end, we observe that at a price vector $p$ with $p_{k}=0$, as good $k$ is free, an agent may purchase an infinite amount of good $k$ even if it does not increase her utility. We will use this freedom of being able to purchase additional quantities of zero-priced goods to modify the definition of $F$ so that it is non-empty, compact and includes non-negative excess demands for the goods with zero prices. In addition, we will extend the domain of $F$ to all of $\mathbb{R}^{n}$ in such a way that the only solutions are those with prices that stay in the domain $\mathbb{R}_{+}^{n}$.

\subsection{Differential Inclusion and Semi-Continuity of Sets}

Definition 1. A differential inclusion is an equation of the form $\frac{d p}{d t} \in F(t, p(t))$, where $F(t, p)$ is a non-empty set for all $t$ and $p$. This generalizes standard differential equations of the form $\frac{d p}{d t}=f(t, p(t))$, where $f(t, p)$ is single-valued.

In our setting, $F$ is a function of $p$ alone.

Let $\mathbb{P}(A)$ denote the power set of the set $A$. Let $\Omega(a)$ denote an open neighborhood of a point $a$.

Definition 2. A set-valued map $F: Z \rightarrow \mathbb{P}(Y)$ is upper semi-continuous at $z_{0} \in Z$ if for any open set $M \in \mathbb{P}(Y)$ which contains $F\left(z_{0}\right)$, there exists $\Omega\left(z_{0}\right)$ such that for all $z \in \Omega\left(z_{0}\right)$, $F(z) \subset M$. A set-valued map $F$ is upper semi-continuous if it is so at every $z_{0} \in Z$.

$A$ set-valued map $F: Z \rightarrow \mathbb{P}(Y)$ is lower semi-continuous at $z_{0} \in Z$ if for any $y_{0} \in F\left(z_{0}\right)$ and any neighborhood $\Omega\left(y_{0}\right)$, there exists a neighborhood $\Omega\left(z_{0}\right)$ such that for all $z \in \Omega\left(z_{0}\right)$, $F(z) \cap \Omega\left(y_{0}\right) \neq \emptyset$. A set-valued map $F$ is lower semi-continuous if it is so at every point $z_{0} \in Z$.

A set-valued map $F: Z \rightarrow \mathbb{P}(Y)$ is continuous at $z_{0} \in Z$ if it is both upper and lower semicontinuous at $z_{0}$. A set-valued map $F$ is continuous if it is so at every $z_{0} \in Z$.

For any sets $A_{1}, A_{2}, \cdots, A_{k}$, let their sumset be $\left\{\sum_{i=1}^{k} a_{i} \mid a_{i} \in A_{i}\right\}$. We state the following basic facts, which will be useful later.

Lemma 8. (a) If $A_{1}, A_{2}, \cdots, A_{k}$ are convex and compact, then their sumset is convex and compact.

(b) If $A_{1}, A_{2}, \cdots, A_{k}: Z \rightarrow \mathbb{P}(Y)$ are upper semi-continuous at $z \in Z$, then their sumset is upper semi-continuous at $z$.

\footnotetext{
${ }^{11}$ An example: in a Leontief Fisher market, it is possible that at an equilibrium some $p_{j}=0$ with a negative excess demand for good $j$.
} 
(c) If $F_{1}, F_{2}: Z \rightarrow \mathbb{P}(Y)$ are two set-valued maps which are upper semi-continuous at $z \in Z$, the map $F^{\cap}: Z \rightarrow \mathbb{P}(Y)$, defined as $F^{\cap}(z)=F_{1}(z) \cap F_{2}(z)$, is also upper semi-continuous at $z \in Z$.

The following Maximum Theorem is well-known in mathematical economics. It provides results on set-valued map semi-continuity, which are among the required conditions for the existence of a solution to our differential inclusions.

Theorem 9 (Maximum Theorem, [3, p. 116]). Let $u: P \times X \rightarrow \mathbb{R}$ be a continuous function, and $C: P \rightarrow \mathbb{P}(X)$ be a compact set-valued map. Let $C^{*}(p)=\arg \max _{x \in C(p)} u(p, x)$ and $u^{*}(p)=\max _{x \in C(p)} u(p, x)$. If $C$ is continuous at some $p$, then $u^{*}$ is continuous at $p$ and $C^{*}$ is non-empty, compact and upper semi-continuous at $p$.

Let $B\left(p_{0}, \rho\right)$ denote the closed ball around $p_{0}$ with radius $\rho$.

Theorem 10 ([28, p. 96-103]). Let $\frac{d p}{d t} \in F(p(t))$ be a differential inclusion, where $F: P \rightarrow$ $\mathbb{P}(\mathbb{R})$ is upper semi-continous at every $p^{\prime} \in B\left(p_{0}, \rho\right)$ for some $\rho>0$. Suppose that $F\left(p^{\prime}\right)$ is convex and compact for every $p^{\prime} \in B\left(p_{0}, \rho\right)$, and there exists a finite $\kappa$ such that $\sup _{\vec{z} \in F\left(p^{\prime}\right)}\|z\| \leq$ $\kappa$ for every $p^{\prime} \in B\left(p_{0}, \rho\right)$. Then for $0 \leq t \leq \rho / \kappa$, there exists an absolutely continuous solution $p(t)$ to the differential inclusion with $p(0)=p_{0}$.

\subsection{Existence of a Solution for (13)}

We will limit the study to the special case where $h$ is a separable function, i.e., it is of the form $\sum_{j} h\left(p_{j}\right)$, for a 1 -dimensional function $h: \mathbb{R} \rightarrow \mathbb{R}$. Now the minimization in (11) separates out into independent minimization problems for each good $j$. We will use $d_{h}\left(p_{j}, q_{j}\right)$ to denote $h\left(p_{j}\right)-h\left(q_{j}\right)-h^{\prime}\left(q_{j}\right)\left(p_{j}-q_{j}\right)$, the one dimensional version of Bregman divergence. Note that as $h$ is convex,

$$
d_{h}\left(p_{j}, q_{j}\right) \geq 0
$$

and, by the strict convexity of $h$,

$$
\text { if } p_{j} \neq q_{j}, \quad d_{h}\left(p_{j}, q_{j}\right)>0 .
$$

As we will see shortly in Lemma 11, $\frac{d p_{j}}{d t}=-\nabla_{j} \phi\left(p^{t}\right) / h^{\prime \prime}\left(p_{j}^{t}\right)$ if $\nabla_{j} \phi\left(p^{t}\right)$ and $h^{\prime \prime}\left(p_{j}^{t}\right)$ are finite. In order to apply Theorem 10 on a ball $B$ around a price vector $p$, we need this term to be bounded on $B$. And, in order to make progress, we will also need that $h^{\prime \prime}\left(p_{j}^{t}\right) \neq \infty$. (Otherwise, we may "get stuck" at a non-equilibrium price since $\frac{d p_{j}}{d t}$ would be 0.) These lead us to make assumptions on the allowable $h$ and on the behavior of the tatonnement, namely that it is controllable, as defined in the subsequent subsections.

\subsubsection{Allowable $h$}

We will also need $h$ to be twice differentiable. It may be that $h^{\prime}(0)=-\infty$, but by the convexity of $h$, this is the only argument for which $h^{\prime}$ might be infinite. And if $h^{\prime}(0)=-\infty$ then $h^{\prime \prime}(0)=\infty$.

Lemma 11. For all $j$, if $\nabla_{j} \phi\left(p^{t}\right)$ and $h^{\prime \prime}\left(p_{j}^{t}\right)$ are finite, then

$$
\lim _{\epsilon \rightarrow 0} \frac{p(\epsilon)-p^{t}}{\epsilon}=\frac{-\nabla_{j} \phi\left(p^{t}\right)}{h^{\prime \prime}\left(p_{j}^{t}\right)} .
$$


Proof. The minimizer in (11) must have a zero derivative:

$$
\nabla_{j} \phi\left(p^{t}\right)+\frac{1}{\epsilon} \frac{d\left(d_{h}\left(p_{j}, p_{j}^{t}\right)\right)}{d p_{j}}=0
$$

Since $\frac{d\left(d_{h}\left(p_{j}, p_{j}^{t}\right)\right)}{d p_{j}}=h^{\prime}\left(p_{j}\right)-h^{\prime}\left(p_{j}^{t}\right)$, substituting in (16) and solving for $p_{j}$ gives

$$
p_{j}(\epsilon)=h^{\prime-1}\left(h^{\prime}\left(p_{j}^{t}\right)-\epsilon \nabla_{j} \phi\left(p^{t}\right)\right) .
$$

Note that since $h$ is strictly convex, $h^{\prime}$ is strictly increasing and hence is invertible. For notational convenience, let $g(y)=h^{-1}(y)$. Then $h^{\prime}(g(y))=y, h^{\prime \prime}(g(y)) \cdot g^{\prime}(y)=1$, therefore $g^{\prime}\left(p_{j}\right)=\frac{1}{h^{\prime \prime}\left(g\left(p_{j}\right)\right)}$. Also note that $g\left(h^{\prime}(y)\right)=y$. Using these we obtain

$$
g^{\prime}\left(h^{\prime}\left(p_{j}\right)\right)=\frac{1}{h^{\prime \prime}\left(p_{j}\right)} .
$$

Strictly speaking, the above argument is not valid for $p_{j}=0$ if $h^{\prime}(0)=-\infty$. But in this case, we can check directly that (17) is still correct, for then $g^{\prime}(-\infty)=0$ and $h^{\prime \prime}(0)=\infty$. Now,

$$
\begin{aligned}
\lim _{\epsilon \rightarrow 0} \frac{p_{j}(\epsilon)-p_{j}^{t}}{\epsilon} & =\lim _{\epsilon \rightarrow 0} \frac{g\left(h^{\prime}\left(p_{j}^{t}\right)-\epsilon \nabla_{j} \phi\left(p^{t}\right)\right)-g\left(h^{\prime}\left(p_{j}^{t}\right)\right)}{\epsilon} \\
& =-g^{\prime}\left(h^{\prime}\left(p_{j}^{t}\right)\right) \cdot \nabla_{j} \phi\left(p^{t}\right) \\
& =-\nabla_{j} \phi\left(p^{t}\right) / h^{\prime \prime}\left(p_{j}^{t}\right) \quad(\text { by }(17)) .
\end{aligned}
$$

We make the following additional assumptions on $h$.

Definition 3. $h(p)$ is allowable if $h$ is twice differentiable and strictly convex (hence $\left.h^{\prime \prime}(p)>0\right)$, $h^{\prime \prime}(p)$ is finite if $p>0,1 / h^{\prime \prime}$ is continuous, and either

A1. The market is a Fisher market, or

A2. $\int_{p}^{\infty} h^{\prime \prime}(q) d q=\infty$ for all $p>0$,

and in addition either

B1. $h^{\prime \prime}(p)$ is finite for all $p$, or

B2. $\int_{0}^{p} h^{\prime \prime}(q) d q=\infty$ for all $p>0$; in this case, we say $h$ is controlling.

Henceforth, we assume that $h$ is allowable.

We note that two of the most commonly used Bregman divergences satisfy the above assumptions. The first one uses $h\left(p_{j}\right)=\frac{1}{2} p_{j}^{2}$; thus $h^{\prime \prime}\left(p_{j}\right)=1$; hence $\frac{d p_{j}}{d t}=-\nabla_{j} \phi(p)$. Also, for $p>0, \int_{p}^{\infty} h^{\prime \prime}(q) d q=\infty$, so conditions A2 and B1 are satisfied. The second one, which is the KL-divergence, uses $h\left(p_{j}\right)=p_{j} \log p_{j}-p_{j}, h^{\prime}\left(p_{j}\right)=\log p_{j}$ and $h^{\prime \prime}\left(p_{j}\right)=1 / p_{j}$. Hence $\frac{d p_{j}}{d t}=-p_{j} \nabla_{j} \phi(p)$. Also, $\int_{0}^{p} \frac{d q}{q}=\log p-\log 0=\infty$ and for $p>0, \int_{p}^{\infty} \frac{d q}{q}=\log \infty-\log p=\infty$, so conditions A2 and B2 are satisfied.

The reason for the condition B2 in Definition 3 is to ensure that if the tatonnement starts at a point with finite $h^{\prime \prime}$ it will never reach a point with infinite $h^{\prime \prime}$. When $h^{\prime \prime}\left(p_{j}\right)=\infty$, by Lemma 11. $\frac{d p_{j}^{t}}{d t}=0$ no matter what the value of $\nabla \phi\left(p^{t}\right)$ is, i.e. $p_{j}$ remains constant hereafter. This is an unreasonable tatonnement rule. 
Lemma 12. Suppose that $h^{\prime \prime}\left(p_{j}^{0}\right)$ is finite. If $h$ is allowable then $h^{\prime \prime}\left(p_{j}^{t}\right)$ is finite for all $t \geq 0$.

Proof. If condition B1 of Definition 3 holds then the result is immediate. So suppose that condition B2 holds. By assumption, $h^{\prime \prime}(p)=\infty$ only if $p=0$. As $z_{j} \geq-1$ always, $\nabla_{j} \phi(p) \leq 1$ always. Consequently, by Lemma $11 \frac{d p_{j}^{t}}{d t} \geq-1 / h^{\prime \prime}\left(p_{j}^{t}\right)$. Suppose that $p_{j}^{0}>0$. Then let $\bar{t}>0$ be the earliest time at which $p_{j}$ could be zero. We use condition B2 to justify the last equality below:

$$
\bar{t} \geq-\int_{0}^{p_{j}^{0}} \frac{d p_{j}^{t}}{d p_{j}^{t} / d t} \geq \int_{0}^{p_{j}^{0}} h^{\prime \prime}(p) d p=\infty .
$$

Thus only at time $t=\infty$ can $p_{j}$ be 0 , and hence only at time $t=\infty$ can $h^{\prime \prime}\left(p_{j}\right)$ be $\infty$.

The reason for the condition A2 in Definition 3 is to ensure that if the tatonnement starts at a point with finite value, no price will blow up to $+\infty$ in finite time.

Lemma 13. Suppose that $p^{0}$ is finite. If $h$ is allowable then $p^{t}$ is finite for all $t \geq 0$.

Proof. If the market is a Fisher market then prices remain bounded by the maximum of their initial value and the amount of money in the market. So suppose the market is not a Fisher market; then, by assumption, $\int_{p}^{\infty} h^{\prime \prime}(q) d q=\infty$ for all $p>0$. Let $p_{\max }=\max p_{j}$. Define $M^{t}=\sum_{j} p_{j}^{t} \leq p_{\max } \cdot n$. Then $z_{\max } \leq n$. So $\frac{d}{d t} p_{\max }^{t} \leq n / h^{\prime \prime}\left(p_{\max }^{t}\right)$.

Let $\bar{t}$ be the earliest time at which $p_{\max }^{t}$ could be infinite. Let tmin $=\arg \min _{t<\bar{t}} p_{\max }^{t}$. If $p_{\max }^{\mathrm{tmin}}>0$, then by Condition A2,

$$
\bar{t} \geq \frac{1}{n} \int_{p_{\max }^{\min }}^{\infty} h^{\prime \prime}(p) d p=\infty,
$$

and if $p_{\max }^{\mathrm{tmin}}=0$, then the same bound holds by Conditions A2 and B2.

The following example shows that a price may blow up to $+\infty$ in finite time if condition A2 is violated.

Example 1. Consider an Arrow-Debreu market with one agent and two goods. The agent has one unit of each good as initial endowment. The agent wants only good 1. So the equilibrium price vector is $\left(p_{1}^{*}, p_{2}^{*}\right)=(p, 0)$ for any $p>0$. At any $\left(p_{1}, p_{2}\right)$, the excess demand for good 1 is $\left(p_{1}+p_{2}\right) / p_{1}-1=p_{2} / p_{1}$ and the excess demand for good 2 is -1 .

Suppose the tatonnement starts at $\left(p_{1}, p_{2}\right)=(2,1)$ and $h$ satisfies $h^{\prime \prime}(p)=1 / p$ for $p \leq 1$ and $h^{\prime \prime}(p)=1 / p^{3}$ for $p \geq 1$. Then $\frac{d p_{2}^{t}}{d t}=-p_{2}^{t}$ and $\frac{d p_{1}^{t}}{d t}=\left(p_{1}^{t}\right)^{2} p_{2}^{t}$. The solution is $p_{1}(t)=\frac{2}{2 e^{-t}-1}$ and $p_{2}(t)=e^{-t}$. Note that $p_{1}(t)$ blows up to $+\infty$ at $t=\log 2$.

\subsubsection{Local Convergence of 13}

Next we show that there is a solution to (13) for some time interval $[0, \bar{t}]$, under additional assumptions. Later, we will show how to extend the solution to arbitrarily large $t$ and remove the assumptions.

In order to apply Theorem 10 to 13$)$, we need its right hand side $\left(-\nabla_{j} \phi(p) / h^{\prime \prime}\left(p_{j}\right)\right.$ when $\nabla_{j} \phi(p)$ is finite) to be convex, compact and upper semi-continuous in any ball $B\left(p_{0}, \rho\right)$ we consider. The difficulty we face is that when some prices are zero, the corresponding demands can be infinite, and then compactness will not hold for such price vectors. 
To restore compactness we modify $F$ as follows. Let $b>0$. We define $F_{b}\left(p^{t}\right)=F\left(p^{t}\right) \cap\{v \mid-$ $b \mathbb{1} \leq v \leq b \mathbb{1}\}$. We then define the following differential inclusion on $\mathbb{R}_{+}^{n}$ :

$$
\frac{d p}{d t}=F_{b}\left(p^{t}\right)
$$

This introduces the possibility that $F_{b}(p)$ is empty for some $p$ which makes the differential inclusion trivially unsatisfiable. We assume for now that $F_{b}(p)$ is non empty in a small neighborhood around $p$, and remove this assumption later.

Definition 4. We say that $F$ is bounded near $p$ if there exists some neighborhood $\Omega(p)$ of $p$ and a finite positive number $b$ such that for all $q \in \Omega(p) \cap \mathbb{R}_{+}^{n}, F_{b}(q)$ is non-empty and $h^{\prime \prime}(q)$ is finite ${ }^{12}$

Lemma 14. Suppose that $h$ is allowable and that $F$ is bounded near $p$. Then $F_{b}(p)$ is convexvalued, compact-valued and upper semi-continuous at $p$.

Proof. Let $\Omega(p)$ be the neighborhood of $p$ given by the assumption that $F$ is bounded near $p$ (Definition 4), and let $B \subset \mathbb{R}_{+}^{n}$ be a compact neighborhood of $p$ such that $B \subset \Omega(p)$ and every positive price in $p$ is positive in $B$. By our choice of $B h^{\prime \prime}\left(q_{j}\right)$ is positive and finite for all $q \in B$ and for all $j$, so there exists a positive number $\bar{h}$ such that $h^{\prime \prime}\left(q_{j}\right) \leq \bar{h}$ for all $q \in B$ and for all $j$. Then on $B, b \geq\left|z_{j}(q) / h^{\prime \prime}\left(q_{j}\right)\right| \geq\left|z_{j}(q) / \bar{h}\right|$, i.e. $x_{j}(q)=z_{j}(q)+1 \leq b \bar{h}+1$. Let $\bar{b}$ denote $b \bar{h}+1$.

We apply Theorem 9 with $P=\Omega(p), X=[0, \bar{b}]^{n} \cdot u$ is the utility function of an agent, which we assume to be continuous and concave. For any $q \in \Omega(p), C(q)$ is the set of all affordable bundles in $X$ of the agent at price $q$. It is well known that $C(q)$ is continuous, and since its range is confined to the compact set $X, C(q)$ is compact-valued. By Theorem 9, $C^{*}(p)$, the set of all affordable optimal bundles of the agent at price $p$ contained in $X$, is compact and upper semi-continuous at $p$. By our assumption that $F_{b}(p)$ is non-empty, $C^{*}(p)$ is also a subset of all affordable optimal bundles of the agent at price $p$ globally (i.e. without confinement to $X$ ). Also, since $u$ is concave, $C^{*}(p)$ is convex.

By the definition of $C^{*}(p)$ and $\phi,-\nabla \phi(p)$ is the sumset of $C^{*}(p)$ over all agents and the set $\{-1\}$. As $C^{*}(p)$ is non-empty for each agent, $-\nabla \phi(p)$ is also non-empty. By Lemma 8(a) and $(\mathrm{b}),-\nabla \phi(p)$ is convex and compact, and it is upper semi-continuous at any $p$. $\left(F_{b}\right)_{j}$ is $-\nabla_{j} \phi(p)$ divided by $h^{\prime \prime}\left(p_{j}\right)$, while $1 / h^{\prime \prime}$ is continuous and positive at any $p \in P$. So the division by $h^{\prime \prime}$ will not affect convexity, compactness and upper semi-continuity.

The following lemma is immediate.

Lemma 15. Any solution to system (18) over time interval $[0, \bar{t}]$ starting at a price vector $p^{0}$ such that $F$ is bounded near $p^{0}$ is also a solution to system (13).

As discussed previously, we want to extend the domain for the differential inclusion to $\mathbb{R}^{n}$. We will work with $F_{b}$ rather than $F$, however. To help specify the new differential inclusion system, for any price vector $p$, we introduce the following notation: letting $p=\left(p_{j}\right)$, we define $p_{j}^{+}=\max \left\{0, p_{j}\right\}$ and $p^{+}=\left(p_{j}^{+}\right)$. The new system is given by

$$
\frac{d p^{t}}{d t} \in G_{b}\left(p^{t}\right)
$$

with $G_{b}$ defined as follows:

\footnotetext{
${ }^{12}$ We remark that this condition is satisfied automatically when $p>\overrightarrow{0}$.
} 
1. For $p \in \mathbb{R}_{+}^{n}, G_{b}(p)=F_{b}(p)$.

2. For $p \notin \mathbb{R}_{+}^{n}$, let $J(p)=\left\{j \mid p_{j}<0\right\}$, then set $G_{b}(p)=G_{b}\left(p^{+}\right) \cap\left\{z \mid \forall j \in J(p), z_{j} \geq 0\right\}$.

Lemma 16. Let $p \in \mathbb{R}^{n}$. Suppose that $h$ is allowable and $F$ is bounded near $p^{+}$. Then $G_{b}(p)$ is convex, compact and upper semi-continuous at $p$.

Proof. As $G_{b} \equiv F_{b}$ in $\mathbb{R}_{+}^{n}$, by Lemma 14 , the result is immediate for $p>\overrightarrow{0}$.

For the other $p$ 's, note that $G_{b}(p)=G_{b}\left(p^{+}\right) \cap\left\{z \mid \forall j \in J(p), z_{j} \geq 0\right\}$ is the intersection of two sets, the first being convex and compact and the second being convex and closed. So $G_{b}(p)$ is convex and compact. What remains is to check upper semi-continuity at these $p$ 's. There are two cases: $p \in \mathbb{R}_{+}^{n}$ but it has some zero prices, or $p \notin \mathbb{R}_{+}^{n}$.

Case 1: $p \in \mathbb{R}_{+}^{n}$ but it has some zero prices. For any open set $M$ which contains $G_{b}(p)=F_{b}(p)$, by Lemma 14, we can take a sufficiently small neighborhood $B(p, \delta)$ of $p$ such that for all $q \in B(p, \delta) \cap \mathbb{R}_{+}^{n}, F_{b}(q) \subset M$. Then, for any $q \in B(p, \delta) \backslash \mathbb{R}_{+}^{n}$, note that $q^{+} \in B(p, \delta)$ since $\left\|q^{+}, p\right\| \leq\|q, p\|$, and, of course, $q^{+} \in \mathbb{R}_{+}^{n}$. Thus $F_{b}\left(q^{+}\right) \subset M$; and $G_{b}(q) \subseteq G_{b}\left(q^{+}\right)=F_{b}\left(q^{+}\right) \subset$ $M$. So $G_{b}$ is upper semi-continuous at $p$.

Case 2: $p \notin \mathbb{R}_{+}^{n}$. For any $q \in \mathbb{R}^{n}$, let $V(q)$ denote the set $\left\{v \mid \forall j \in J(q), v_{j} \geq 0\right\}$. For any $q \notin \mathbb{R}_{+}^{n}, G_{b}(q)=G_{b}\left(q^{+}\right) \cap V(q)$. By Case 1 and our conditions on $p, G_{b}\left(p^{+}\right)$is upper semi-continuous at $p^{+}$. $p^{+}$is continuous in $p$. Hence $G_{b}\left(p^{+}\right)$is upper semi-continuous at $p$. Next, we observe that there exists a small $\delta>0$ such that for all $q \in B(p, \delta)$, if $p_{j} \neq 0$, then $\operatorname{sign}\left(q_{j}\right)=\operatorname{sign}\left(p_{j}\right)$ and consequently $V(q) \subseteq V(p)$; it immediately follows that $V(p)$ is upper semi-continuous at $p$. Now, by Lemma $8(c), G_{b}(p)$ is upper semi-continuous at $p$.

Lemma 17. Any solution to system (19) over time interval $[0, \bar{t}]$ starting at price vector $p^{0}$ is also a solution of (13) if $F$ is bounded near $p^{0}$.

Proof. We will show that any solution of $(19)$ is a solution of (18). The result then follows from Lemma 15.

In the definition of $G_{b}$, at a price vector $p$ with $p_{j}<0, G_{b, j}(p)$ is always positive or zero, so it is impossible for any tatonnement trajectory satisfying $(19)$ to enter the region $p_{j}<0$. Hence, all prices remain positive or zero, i.e. $p^{t} \in \mathbb{R}_{+}^{n}$ for all $t$. In $\mathbb{R}_{+}^{n},(18)$ is identical to (19), so we are done.

Lemma 18. Suppose that $h$ is allowable, and $F$ is bounded near $p^{0}$. Then there is a time $\bar{t}>0$ such that (13) has an absolutely continuous solution for time interval $[0, \bar{t}]$ with $p(0)=p^{0}$.

Proof. By Lemma 16, $G_{b}(p)$ is convex, compact and upper semi-continuous at $p$ in the interior of $\Omega\left(p^{0}\right)$. Now, by Theorem 10, (19) has an absolutely continuous solution with $p(0)=p^{0}$ for some time interval $[0, \bar{t}]$, where $\bar{t}>0$. And by Lemma 17, this is also a solution to (13).

Lemma 18 gives us a local solution (i.e., upto some time $\bar{t}>0$ ) under the assumption that $F$ is bounded near $p^{0}$. We need to remove this assumption and we need a solution for arbitrarily large $\bar{t}$. For these we need the notion of controllability. 


\subsubsection{Controllability}

Given a starting price vector $p^{0}$ and any finite time $\bar{t} \geq 0$, we need to ensure that there is a sufficiently large $b=b\left(p^{0}, \bar{t}\right)$ guaranteeing that the tatonnement remains in the domain with $F_{b} \neq \phi$ during the time interval $[0, \bar{t}]$ (so that the differential inclusion is defined for all points encountered during the tatonnement). This will be ensured by the assumption of controllability. To understand this, we first need to characterize the set of optimal bundles of an agent at price vector $p$. There are two possibilities:

1. Every bundle includes at least one good having infinite demand. Then we say that $p$ is an unbounded demand price vector. Note that this good must then have price zero, and by Lemma 12 this can occur only if $h^{\prime \prime}(0)$ is finite. Via the controllability requirement, we will ensure that in this case the tatonnement trajectory does not reach any unbounded demand price vector. (If $h^{\prime \prime}(0)$ is infinite then this is already ensured by Lemma 12).

2. All the demands in at least one bundle are finite. Then we say that $p$ is a bounded demand price vector. Note that if $p$ includes a zero price, $p_{j}=0$ say, then an optimal bundle can have an infinite demand for good $j$; but $p$ is a bounded demand price vector if for all such $j$, the demand for good $j$ could be finite.

For instance, in a Leontief Fisher market, an equilibrium price vector may include a zero price but it will be a bounded demand price vector; clearly, we want the tatonnement trajectory to be able to converge to it. Furthermore, in this case, as the tatonnement proceeds, we want the agent's sequence of optimal bundles to always have bounded demands, and further these bounds should apply throughout the tatonnement process.

We are now ready to define controllability.

Definition 5. Let $\phi$ be a potential function and $\mathcal{T}$ a continuous tatonnement rule. The pair $(\phi, \mathcal{T})$ is controlled, if for any bounded demand starting price vector $p^{0}$ and any finite time $\bar{t} \geq 0$, there are finite bounds $b\left(p^{0}, \bar{t}\right)$ and $c\left(p^{0}, \bar{t}\right)$ such that for any tatonnement trajectory induced by (18), there exists a neighborhood $\Omega$ of the trajectory in which for any $p \in \Omega$ and for any $j$,

1. $\left|-\nabla_{j} \phi(p) / h^{\prime \prime}\left(p_{j}\right)\right| \leq b\left(p^{0}, \bar{t}\right)$ and $p \leq c\left(p^{0}, \bar{t}\right)$ for all $0 \leq t \leq \bar{t}$;

2. $\lim _{t \nearrow \bar{t}} b\left(p^{0}, t\right)$ and $\lim _{t \nearrow \bar{t}} c\left(p^{0}, t\right)$ are finite 13 ,

i.e. both the prices and the rate of change of the prices remain bounded throughout the tatonnement process up to and including time $\bar{t}$.

We will show that if $h$ is controlling (recall Definition 3) then $(\phi, \mathcal{T})$ is controlled. We will also show that controllability is obeyed by Fisher markets with CES, Leontief and linear utilities along with any tatonnement rule (i.e. even if $h$ is not controlling). But it is not clear if the latter result applies to all markets or even to all EG markets. One example for which we have not resolved this question are Fisher markets with nested CES utilities (see [22] for a definition); these are EG markets.

\footnotetext{
${ }^{13}$ Without loss of generality, we may assume that $b\left(p^{0}, t\right), c\left(p^{0}, t\right)$ are increasing functions of $t$, so the limits exist.
} 
Lemma 19. If $h$ is controlling then $(\phi, \mathcal{T})$ is controlled.

Proof. As $h$ is controlling, in finite time $\bar{t}$, the trajectory is both upper-bounded and bounded away from zerq ${ }^{14}$, say $0<\underline{p}(\bar{t}) \leq p_{j}^{t} \leq \bar{p}(\bar{t})<+\infty$, for all $j$ and for all $0 \leq t \leq \bar{t}$. Then there exists a neighborhood $\Omega$ of the trajectory up to time $\bar{t}$ such that all prices in $\Omega$ are between $p(\bar{t}) / 2$ and $\bar{p}(\bar{t})+1$. Set $c\left(p^{0}, \bar{t}\right)=\bar{p}(\bar{t})+1$.

For all $p \in \Omega, 0<\underline{p}(\bar{t}) / 2 \leq p_{j} \leq \bar{p}(\bar{t})+1<+\infty$, so $h^{\prime \prime}\left(p_{j}\right)$ is bounded away from 0 .

As $\phi$ is convex, $\nabla \phi$ is finite except possibly at the boundary, i.e. when one or more prices is zero. When all prices are between $p(\bar{t}) / 2$ and $\bar{p}(\bar{t})+1, \nabla \phi$ is bounded. Combined with the last paragraph, $\left|-\nabla \phi(p) / h^{\prime \prime}\left(p_{j}\right)\right|$ is bounded on $\Omega$. Set $b\left(p^{0}, \bar{t}\right)$ to be an upper bound of $\left|-\nabla \phi(p) / h^{\prime \prime}\left(p_{j}\right)\right|$ on $\Omega$.

The proof of the next lemma is in the appendix.

Lemma 20. Fisher markets with CES, Leontief and linear utilities along with any tatonnement rule are all controlled.

Now we are ready to complete the proof of the existence of a solution to the differential inclusion system (13).

Lemma 21. Suppose that $h^{\prime \prime}\left(p^{0}\right)$ is finite, $h$ is allowable and $(\phi, \mathcal{T})$ is controlled. Then for any bounded demand starting price vector $p^{0}$ there exists a solution $p^{t}$ to 13 for time range $[0, \infty)$, with $p^{t}$ an absolutely continuous function for any bounded time span, and $p^{t}(t=0)=p^{0}$.

Proof. We will prove the result for differential inclusion $(19)$ and then the result follows from Lemma 17.

The controllability assumption allows us to pick $b=b\left(p^{0}, t\right)$ for some $t>0$ and have $F$ be bounded near $p$. We can therefore apply Lemma 18 to get a solution for some time interval $\left[0, t^{\prime}\right]$ with $t^{\prime}>0$. By Lemma 17, this is also a solution for (13). Once again, due to the assumption of controllability, the solution path cannot end at a point with $\nabla_{j} \phi / h^{\prime \prime}=-\infty$ for any $j$. By the continuity of $\nabla_{j} \phi / h^{\prime \prime}$, there is then a ball around $p^{t^{\prime}}$ in which $\nabla_{j} \phi / h^{\prime \prime}$ is bounded. So we can repeatedly extend the path by additional applications of Lemma 18. Suppose that this yields an open path ending at but possibly not reaching some time $\bar{t}$. We first argue that it can be extended to $\bar{t}$ and then can be extended yet further.

By the controllability assumption, for any $t \in[0, \bar{t})$, all prices in $p^{t}$ are bounded by $\lim _{t} \bar{t}_{t} c\left(p^{0}, t\right)$, which is finite; then the sequence $\left\{p^{t}\right\}_{0 \leq t<\bar{t}}$ has a cluster point $\tilde{p}$. Then by the controllability assumption again, all $\frac{d p_{j}^{t}}{d t}$ are bounded by $\lim _{t} \bar{t}_{t} b\left(p^{0}, t\right)$, which is again finite. Hence, $\left\{p^{t}\right\}_{0 \leq t<\bar{t}}$ has at most one cluster point. So $\tilde{p}$ is the unique cluster point of the sequence $\left\{p^{t}\right\}_{0 \leq t<\bar{t}}$. Setting $p^{t}(t=\bar{t})=\tilde{p}$ extends the solution to $t=\bar{t}$.

Again by the controllability assumption, there exists a neighborhood of $\tilde{p}$ such that all $q$ in the neighborhood has finite $\nabla \phi(q) / h^{\prime \prime}(q)$. By Lemma 18 , we can extend the path $P$ beyond time $\bar{t}$ by at least a positive time period.

\footnotetext{
${ }^{14}$ These follow easily from the proofs of Lemma 12 and Lemma 13
} 


\subsection{Differential inclusion (13) converges}

In Arrow-Debreu markets, it is well-known that if $p^{*}$ is an equilibrium price vector, then $c p^{*}$, where $c$ is any positive constant, is also an equilibrium price vector. It is standard to consider normalized prices, price vectors $\hat{p}$ such that $\sum \hat{p}=1$. Note that for any price vector $p$ with at least one positive price, the corresponding normalized price vector $\hat{p}$ is given by $\hat{p}_{j}=p_{j} /\left(\sum_{\ell} p_{\ell}\right)$.

We are ready to state the main result of this section.

Theorem 22. Let $\phi: \mathbb{R}_{+}^{n} \rightarrow \mathbb{R}$ and $p^{t} \in \mathbb{R}_{+}^{n}$ be defined by 11)-(13). Suppose that $\phi$ is convex and $h$ is allowable. Further suppose that $\phi$ together with the tatonnement rule given by (13) is controlled. Then, for any starting bounded demand price vector $p^{0}$ such that for all $j, h^{\prime \prime}\left(p_{j}^{0}\right)$ is finite, if the market is a Fisher market, then

$$
\lim _{t \rightarrow \infty} p^{t}=p^{*}
$$

where $p^{*}$ is a minimizer of $\phi$.

In Arrow-Debreu markets, if in addition $d_{h}$ is the KL-divergence then

$$
\lim _{t \rightarrow \infty} \hat{p}^{t}=\hat{p}^{*}
$$

where $\hat{p}^{*}$ is a normalized minimizer of $\phi$.

For any CPF market, by definition, there exists a $\phi$ such that $-\nabla \phi(p)=z(p)$. Substituting $z$ for $-\nabla \phi$ in (11) - 13) gives a tatonnement update rule for which, by Theorem 22 , the potential converges to its equilibrium value.

Lemma 23. For any Arrow-Debreu market in which $\phi$ exists, for any positive real number $c$, $\phi(p)=\phi(c p)$.

Proof. By Walras law, $p \cdot \nabla \phi(p)=0$. By the definition of $\phi, \nabla \phi(p)=\nabla \phi(c p)$. By the definition of subgradient,

$$
\phi(p) \geq \phi(c p)+(p-c p) \cdot \nabla \phi(c p)=\phi(c p)+(1-c) p \cdot \nabla \phi(p)=\phi(c p)
$$

and

$$
\phi(c p) \geq \phi(p)+(c p-p) \cdot \nabla \phi(p)=\phi(p)+(c-1) p \cdot \nabla \phi(p)=\phi(p) .
$$

These two inequalities imply that $\phi(p)=\phi(c p)$.

Lemma 24. Suppose that $h$ is allowable and $h^{\prime \prime}\left(p_{j}^{0}\right)$ is finite for all $j$. Let $p^{*}$ be any minimizer of $\phi$. Then $d_{h}\left(p_{j}^{*}, p_{j}^{t}\right)$ is finite for all $t$ and $j$.

Suppose that $\phi$ is the potential function for a Fisher market. Then $\sum_{j} \frac{d}{d t} d_{h}\left(p_{j}^{*} ; p_{j}^{t}\right)<0$, unless $p^{t}$ is a minimizer of $\phi$.

Suppose that $\phi$ is the potential function for an Arrow-Debreu market. Then let $\hat{p}^{*}$ be any normalized minimizer of $\phi$, and suppose that $d_{h}$ is the $K L$-divergence. Then $\hat{p}^{t}$, the normalized price vector corresponding to $p^{t}$, satisfies $\sum_{j} \frac{d}{d t} d_{h}\left(\hat{p}_{j}^{*}, \hat{p}_{j}^{t}\right)<0$, unless $p^{t}$ is a minimizer of $\phi$.

Proof. By Lemma 12, $h^{\prime \prime}\left(p_{j}^{t}\right)$ is finite for all $t$ and $j$, and hence so is $h^{\prime}\left(p_{j}^{t}\right)$. As $h$ is always finite, it follows that $d_{h}\left(p_{j}^{*}, p_{j}^{t}\right)=h\left(p_{j}^{*}\right)-h\left(p_{j}^{t}\right)-h^{\prime}\left(p_{j}^{t}\right)\left(p_{j}^{*}-p_{j}^{t}\right)$ is finite. 
To avoid clutter we write $p_{j}$ for $p_{j}^{t}$. We first prove the result for Fisher markets. Recall that $d_{h}\left(p_{j}^{*}, p_{j}\right)=h\left(p_{j}^{*}\right)-h\left(p_{j}\right)-h^{\prime}\left(p_{j}\right)\left(p_{j}^{*}-p_{j}\right)$. So,

$$
\begin{aligned}
\frac{d}{d t} d_{h}\left(p_{j}^{*}, p_{j}\right) & =-\frac{d h\left(p_{j}\right)}{d t}-\frac{d h^{\prime}\left(p_{j}\right)}{d t}\left(p_{j}^{*}-p_{j}\right)+h^{\prime}\left(p_{j}\right) \frac{d p_{j}}{d t} \\
& =-h^{\prime \prime}\left(p_{j}\right) \cdot \frac{d p_{j}}{d t} \cdot\left(p_{j}^{*}-p_{j}\right) \quad\left(\text { since } \frac{d h\left(p_{j}\right)}{d t}=h^{\prime}\left(p_{j}\right) \frac{d p_{j}}{d t}\right) \\
& =\nabla_{j} \phi(p) \cdot\left(p_{j}^{*}-p_{j}\right) \quad(\text { from Lemma 11). }
\end{aligned}
$$

By the definition of the subgradient, $\phi\left(p^{*}\right) \geq \phi(p)+\nabla \phi(p) \cdot\left(p^{*}-p\right)$. Thus

$$
\sum_{j} \frac{d}{d t} d_{h}\left(p_{j}^{*}, p_{j}\right)=\sum_{j} \nabla_{j} \phi(p) \cdot\left(p_{j}^{*}-p_{j}\right) \leq \phi\left(p^{*}\right)-\phi(p)<0,
$$

unless $p=p^{*}$.

Next we prove the result for Arrow-Debreu markets. Let $S=\sum_{\ell} p_{\ell}$. Then $\hat{p}_{j}=p_{j} / S$.

$$
\begin{aligned}
& \frac{d}{d t} d_{h}\left(\hat{p}^{*}, \hat{p}\right) \\
= & \sum_{j} \frac{\partial d_{h}\left(\hat{p}_{j}^{*}, \hat{p}_{j}\right)}{\partial \hat{p}_{j}} \cdot \frac{\partial \hat{p}_{j}}{\partial t}=\sum_{j} \frac{\partial d_{h}\left(\hat{p}_{j}^{*}, \hat{p}_{j}\right)}{\partial \hat{p}_{j}} \sum_{k} \frac{\partial \hat{p}_{j}}{\partial p_{k}} \cdot \frac{\partial p_{k}}{\partial t} \\
= & \sum_{j} \frac{\partial d_{h}\left(\hat{p}_{j}^{*}, \hat{p}_{j}\right)}{\partial \hat{p}_{j}}\left[\frac{1}{S} \frac{\partial p_{j}}{\partial t}+\sum_{k} \frac{-p_{j}}{S^{2}} \frac{\partial p_{k}}{\partial t}\right] \\
= & \frac{1}{S^{2}} \sum_{j} h^{\prime \prime}\left(\hat{p}_{j}\right) \cdot\left(\hat{p}_{j}^{*}-\hat{p}_{j}\right)\left[S \frac{\nabla_{j} \phi(p)}{h^{\prime \prime}\left(p_{j}\right)}-p_{j} \sum_{k} \frac{\nabla_{k} \phi(p)}{h^{\prime \prime}\left(p_{k}\right)}\right] \\
= & \frac{1}{S} \sum_{j} \frac{h^{\prime \prime}\left(\hat{p}_{j}\right)}{h^{\prime \prime}\left(p_{j}\right)} \nabla_{j} \phi(p) \cdot\left(\hat{p}_{j}^{*}-\hat{p}_{j}\right)-\frac{1}{S^{2}}\left(\sum_{k} \frac{\nabla_{k} \phi(p)}{h^{\prime \prime}\left(p_{k}\right)}\right) \sum_{j} p_{j} h^{\prime \prime}\left(\hat{p}_{j}\right) \cdot\left(\hat{p}_{j}^{*}-\hat{p}_{j}\right) .
\end{aligned}
$$

When $h$ is the kernel of the KL-divergence, $h^{\prime \prime}\left(\hat{p}_{j}\right)=\frac{1}{\hat{p}_{j}}=\frac{S}{p_{j}}$. Thus $p_{j} h^{\prime \prime}\left(\hat{p}_{j}\right)=S$ and $\frac{h^{\prime \prime}\left(\hat{p}_{j}\right)}{h^{\prime \prime}\left(p_{j}\right)}=S$. It follows that

$$
\frac{d}{d t} d_{h}\left(\hat{p}^{*}, \hat{p}\right)=\sum_{j} \nabla_{j} \phi(p) \cdot\left(\hat{p}_{j}^{*}-\hat{p}_{j}\right)-\frac{1}{S}\left(\sum_{k} \frac{\nabla_{k} \phi(p)}{h^{\prime \prime}\left(p_{k}\right)}\right)\left(\sum_{j}\left(\hat{p}_{j}^{*}-\hat{p}_{j}\right)\right) .
$$

Since $\hat{p}^{*}$ and $\hat{p}$ are both normalized prices, the second term on the RHS is zero. Noting that $\nabla_{j} \phi(p)=\nabla_{j} \phi(\hat{p})$, and by Lemma 23 , we see that the rest of the argument is the same as for the Fisher markets.

Proof of Theorem 22. In a Fisher market the prices will be bounded by the maximum of their initial values and $\sum_{i} e_{i}$. In an Arrow-Debreu market, we consider only the normalized prices, and these too are bounded. Let $B$ denote the bounded set of prices. We may assume that $B$ is closed 15

The proof comprises four steps:

1. As $p^{t}$ lies in a bounded domain, it must have a convergent subsequence, which converges to a point $q$, say.

\footnotetext{
${ }^{15}$ If not, replace $B$ by its closure.
} 
2. Let $P^{*}$ denote the set of equilibrium prices for Fisher markets, or the set of normalized equilibrium prices for Arrow-Debreu markets. Recall that $d_{h}\left(p^{*}, p\right)=\sum_{j} d_{h}\left(p_{j}^{*}, p_{j}\right)$. Then, for any fixed $p^{*} \in P^{*}$, we can conclude from Lemma 24 that $d_{h}\left(p^{*} ;, p^{t}\right)$ is monotonically decreasing. By (14), $d_{h}\left(p^{*} ;, p^{t}\right) \geq 0$; consequently $\lim _{t \rightarrow \infty} d_{h}\left(p^{*}, p^{t}\right)$ exists, and it must equal $d_{h}\left(p^{*} ; q\right)$, by the continuity of $d_{h}$.

3. Show that $q$ is a minimizer of $\phi$. (Proof below.)

4. By the second and the third steps, $d_{h}\left(q ;, p^{t}\right) \rightarrow d_{h}(q, q)=0$. Using this, show that $p^{t} \rightarrow q$. (Proof below.)

Proof of Step 3. Suppose that $q$ were not a minimizer of $\phi$.

Note that the set $P^{*}$ is closed (due to the continuity of $\phi$ ), so $P^{*} \cap B$ is compact. Let $d\left(q^{\prime}\right)=\min _{p^{\prime} \in P^{*} \cap B} d_{h}\left(p^{\prime}, q^{\prime}\right)$; since $P^{*} \cap B$ is compact, the minimum is attained.

Since $q \notin P^{*}, d(q)>0$. Also, by Lemma $24, d_{h}\left(p^{*}, q\right)$ is finite, hence $d(q) \leq d_{h}\left(p^{*}, q\right)$ is also finite. Let $Q=\left\{q^{\prime} \mid d\left(q^{\prime}\right) \geq d(q)\right\} \cap B$. Since $d_{h}$ is continuous and $P^{*} \cap B$ is compact, it follows that $Q$ is compact. Let $\delta=\min _{q^{\prime} \in Q} \phi\left(q^{\prime}\right)-\phi\left(p^{*}\right)$, since $Q$ is compact, the minimum is attained. By definition, $Q$ contains no minimizer of $\phi$, so $\delta>0$.

From Step 2, for any $p^{*} \in P^{*} \cap B$, for all $t \geq 0, d_{h}\left(p^{*}, p^{t}\right) \geq d_{h}\left(p^{*}, q\right)$ and $d_{h}\left(p^{*}, q\right) \geq d(q)$, so $p^{t} \in Q$ for all $t \geq 0$. By (20), $\frac{d}{d t} d_{h}\left(p^{*}, p^{t}\right) \leq-\left[\phi\left(p^{t}\right)-\phi\left(p^{*}\right)\right] \leq-\delta<0$, which implies that $d_{h}\left(p^{*}, p^{t}\right)$ will eventually go below zero, a contradiction.

Proof of Step 4. Suppose that $p^{t}$ does not converge to $q$. Then there exists an $\epsilon>0$ such that

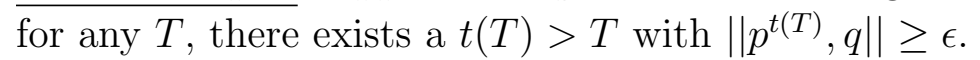

Let $A=\{p \mid\|q, p\| \geq \epsilon\}$, which is closed. Note that $A \cap B$ is compact. Since $d_{h}(q ; p)$ is nonnegative (but possibly $+\infty$ ), finite at some $p \in A \cap B$ (e.g. $p^{t(T)}$ for any $T$ ), and continuous at every $p \in A \cap B$ at which it is finite, $\inf _{p \in A \cap B} d_{h}(q ; p)=\min _{p \in A \cap B} d_{h}(q ; p)=\delta^{\prime}>0$, by (15). Since $p^{t(T)} \in A \cap B, d_{h}\left(q, p^{t(T)}\right) \geq \min _{p \in A \cap B} d_{h}(q ; p)=\delta^{\prime}>0$, i.e. $d_{h}\left(q ; p^{t}\right)$ does not converge to zero, a contradiction.

\section{$5 \quad$ Leontief Utilities}

In this section we consider Fisher markets in which every buyer has a Leontief utility. We analyze the update rule (6) with $d_{h}=6 \cdot \gamma \cdot d_{\mathrm{KL}}$ where $d_{\mathrm{KL}}$ is the KL-divergence, and $\gamma$ is a market dependent parameter. This update rule, which is minimizing $\nabla \phi\left(p^{t}\right) \cdot\left(p-p^{t}\right)+\gamma d_{h}\left(p, p^{t}\right)$ or equivalently is minimizing $-z \cdot\left(p-p^{t}\right)+\gamma\left[p \log p-p-\log p^{t} \cdot\left(p-p^{t}\right)\right]$, amounts to

$$
p_{j}^{t+1}=p_{j}^{t} \exp \left(z_{j} / \gamma\right)
$$

We show an $O(1 / \epsilon)$ convergence rate as specified in the next theorem.

Theorem 25. For a Leontief market, for a sequence of price updates defined by (21), for all $t$,

$$
\phi\left(p^{t}\right)-\phi\left(p^{*}\right) \leq \frac{6 \gamma d_{K L}\left(p^{*}, p^{0}\right)}{t}
$$

where $\gamma=5 \cdot \max _{j}\left\{x_{j}^{\circ}+2 \cdot \sum_{i} \max _{k} \frac{b_{i j}}{b_{i k}}\right\}$. 
The theorem follows by showing that the sandwiching property (9) required by Theorem 2 is satisfied, which is done in Lemma 27 below (recall that $d_{h}=6 \cdot \gamma \cdot d_{\mathrm{KL}}$ here).

We also show that in general the convergence rate is $\Omega(1 / \sqrt{\epsilon})$ as specified in the next theorem (the proof of this result can be found in the appendix).

Theorem 26. There is a 2-good, 2-buyer Leontief market such that

$$
\phi\left(p^{t}\right)-\phi\left(p^{*}\right)=\Omega\left[\frac{\phi\left(p^{0}\right)-\phi\left(p^{*}\right)}{t^{2}}\right] .
$$

By Lemma 7, $\phi\left(p^{t}\right)=\sum_{j} p_{j}^{t}-\sum_{i} e_{i} \log \nu_{i}$, where $\nu_{i}$ is the minimum cost buyer $i$ has to pay to obtain one unit of utility. By (2) the maximum utility obtainable by buyer $i$ equals $e_{i} / \sum_{j} b_{i j} p_{j}$. This utility is obtained by spending $e_{i}$ money; consequently, the minimum cost for one unit of utility is $\sum_{j} b_{i j} p_{j}$. Thus the potential function is given by

$$
\phi\left(p^{t}\right)=\sum_{j} p^{t}-\sum_{i} e_{i} \log \sum_{j} b_{i j} p_{j}
$$

Notation We let $x^{t}$ denote the demands following the price update at time $t$, and $x^{\circ}$ denote the initial demands. We also let $\Delta p_{j}=p_{j}^{t+1}-p_{j}^{t}$ for all $j$.

Lemma 27. If $\left|\Delta p_{j}\right| \leq p_{j} / 4$, then

$$
\phi\left(p^{t+1}\right)-\ell_{\phi}\left(p^{t+1} ; p^{t}\right) \leq 6 \gamma d_{K L}\left(p^{t+1}, p^{t}\right) .
$$

Thus the sandwiching property (9) holds if $\left|\Delta p_{j}\right| \leq p_{j} / 4$. To ensure this, we require that $\gamma \geq 5 \cdot \max _{j, t}\left\{1, x_{j}^{t}\right\}$, where we are maximizing the $x_{j}^{t}$ over all the time steps of the algorithm, for then $p_{j}^{t+1} \leq p_{j}^{t} e^{1 / 5}$ and $\left|\Delta p_{j}\right| / p_{j} \leq e^{1 / 5}-1 \leq \frac{1}{4}$. Of course, $\gamma$ has to be picked at the beginning, at which point one may not know the value of $\max _{j, t}\left\{1, x_{j}^{t}\right\}$. In the following lemma, we show that picking $\gamma=5 \cdot \max _{j}\left\{x_{j}^{\circ}+2 \cdot \sum_{i} \max _{k} \frac{b_{i j}}{b_{i k}}\right\}$ suffices. However, if a better bound were known, that could be used instead.

Proof. (of Theorem 25.) The result follows by applying Theorem 2. To do this, it suffices to ensure that Equation (9) holds for every price update. This is guaranteed by Lemma 27, for, as we have just seen, by construction $\left|\Delta p_{j}\right| \leq p_{j} / 4$ for every price update.

Lemma 28. For any continuous tatonnement, $x_{j}^{t} \leq x_{j}^{\circ}+\sum_{i} \max _{k} \frac{b_{i j}}{b_{i k}}$, and for the discrete tatonnement with update rule (21), $x_{j}^{t} \leq x_{j}^{\circ}+2 \cdot \sum_{i} \max _{k} \frac{b_{i j}}{b_{i k}}$, for all goods $j$ and all times $t$.

Proof. We drop the superscript $t$ when the meaning is clear from the context. Suppose that $x_{i j}=e_{i} \cdot b_{i j} / \sum_{k} b_{i k} p_{k} \geq 1$; then $x_{j} \geq 1$ and so $p_{j}$ can only increase. If $\min _{l} e_{i} \cdot b_{i \ell} / \sum_{k} b_{i k} p_{k} \geq 1$, or equivalently if $e_{i} / \sum_{k} b_{i k} p_{k} \geq 1 / \min _{l} b_{i \ell}$, then every $p_{k}$ for which $b_{i k} \neq 0$ can only increase, and hence the $x_{i k}$ for which $b_{i k} \neq 0$ can only decrease; i.e. if $x_{i j}=e_{i} \cdot b_{i j} / \sum_{k} b_{i k} p_{k} \geq$ $b_{i j} / \min _{l} b_{i \ell}=\max _{k} b_{i j} / b_{i k}, x_{i j}$ can only decrease. Hence, for any continuous tatonnement, $x_{i j}$ is never larger than the maximum of this value and its initial value; i.e. $x_{i j} \leq \max _{k}\left\{x_{i j}^{\circ}, b_{i j} / b_{i k}\right\}$. Thus, in this case, $x_{j}^{t} \leq x_{j}^{\circ}+\sum_{i} \max _{k} \frac{b_{i j}}{b_{i k}}$. In the case of the discrete price updates, in one round of price changes, the prices drop by at most $\exp \left(\frac{1}{5}\right)$, and hence the demands increase by at most $\exp \left(\frac{1}{5}\right) \leq 2$. Thus, unless initially larger, $x_{i j}<2 \cdot \max _{k} b_{i j} / b_{i k}{ }^{16}$. Thus $x_{i j} \leq \max _{k}\left\{x_{i j}^{\circ}, 2 \cdot b_{i j} / b_{i k}\right\}$. Consequently, $x_{j}=\sum_{i} x_{i j} \leq x_{j}^{\circ}+2 \cdot \sum_{i} \max _{k} \frac{b_{i j}}{b_{i k}}$.

\footnotetext{
${ }^{16} \mathrm{~A}$ more careful argument shows the multiplier of 2 is not needed.
} 
Before proving Lemma 27, we state the following claims, proved in the appendix. We let $\Delta p_{j}$ denote $p^{t+1}-p^{t}$. In the following claims, the index $t$ on the prices and demands is implicit.

Claim 29. For all $j$,

$$
\frac{1}{e_{i}} \sum_{j, k} x_{i j} x_{i k}\left|\Delta p_{j}\right| \cdot\left|\Delta p_{k}\right| \leq \sum_{l} \frac{x_{i \ell}}{p_{\ell}}\left(\Delta p_{\ell}\right)^{2} .
$$

Claim 30. Suppose that for all $j,\left|\Delta p_{j}\right| \leq p_{j} / 4$. Then

$$
\frac{\left(\Delta p_{j}\right)^{2}}{p_{j}} \leq \frac{9}{2} d_{K L}\left(p_{j}+\Delta p_{j}, p_{j}\right)
$$

Proof of Lemma 27. We write $\phi\left(p^{t}\right)$ and $\phi\left(p^{t+1}\right)$ as functions of the $p_{j}$, and then upper bound these terms using the inequalities $x(1+x)^{-1} \leq x+\frac{4}{3} x^{2}$ for $|x| \leq \frac{1}{4}$ and $\log (1+y) \leq y$ for $|y| \leq 1$, along with Claims 29 and 30 .

$$
\begin{aligned}
& \phi\left(p^{t+1}\right)-\ell_{\phi}\left(p^{t+1} ; p^{t}\right) \\
= & \phi\left(p^{t+1}\right)-\phi\left(p^{t}\right)-\nabla \phi\left(p^{t}\right) \cdot\left(p^{t+1}-p^{t}\right) \\
= & \sum_{j}\left(p_{j}+\Delta p_{j}\right)-\sum_{i} e_{i} \log \sum_{k} b_{i k}\left(p_{k}+\Delta p_{k}\right)-\sum_{j} p_{j}+\sum_{i} e_{i} \log \sum_{k} b_{i k} p_{k}+\sum_{j} z_{j} \Delta p_{j} \\
= & \sum_{j} x_{j} \Delta p_{j}+\sum_{i} e_{i} \log \frac{\sum_{k} b_{i k} p_{k}}{\sum_{k} b_{i k}\left(p_{k}+\Delta p_{k}\right)} \\
= & \sum_{j} x_{j} \Delta p_{j}+\sum_{i} e_{i} \log \left[1-\frac{\sum_{k} b_{i k} \Delta p_{k}}{\sum_{k} b_{i k} p_{k}}\left(1+\frac{\sum_{l} b_{i \ell} \Delta p_{\ell}}{\sum_{l} b_{i \ell} p_{\ell}}\right)^{-1}\right] .
\end{aligned}
$$

Next we use the bound $x(1+x)^{-1} \leq x+\frac{4}{3} x^{2}$ for $|x| \leq \frac{1}{4}$, noting that $\left|\frac{\sum_{l} b_{i \ell} \Delta p_{\ell}}{\sum_{l} b_{i k} p_{\ell}}\right| \leq \frac{1}{4}$, as every $\left|\Delta p_{\ell}\right| \leq \frac{1}{4} p_{\ell}$ by assumption. Thus:

$$
\phi\left(p^{t+1}\right)-\ell_{\phi}\left(p^{t+1}\right) \leq \sum_{j} x_{j} \Delta p_{j}+\sum_{i} e_{i} \log \left[1-\frac{\sum_{k} b_{i k} \Delta p_{k}}{\sum_{k} b_{i k} p_{k}}+\frac{4}{3} \frac{\sum_{k} b_{i k} \Delta p_{k} \sum_{l} b_{i \ell} \Delta p_{\ell}}{\sum_{k} b_{i k} p_{k} \sum_{l} b_{i \ell} p_{\ell}}\right] .
$$

Now we use the bound $\log (1+y) \leq y$, which applies as the second and third terms in the $\log$ are each bounded by $\frac{1}{4}$ (note that $\left|\frac{\sum_{l} b_{i \ell} \Delta p_{\ell}}{\sum_{l} b_{i k} p_{\ell}}\right| \leq \frac{1}{4}$ ). Hence:

$$
\begin{aligned}
& \phi\left(p^{t+1}\right)-\ell_{\phi}\left(p^{t+1}\right) \\
\leq & \sum_{j} x_{j} \Delta p_{j}-\sum_{i} e_{i} \frac{\sum_{k} b_{i k} \Delta p_{k}}{\sum_{k} b_{i k} p_{k}}+\frac{4}{3} e_{i} \frac{\sum_{k} b_{i k} \Delta p_{k} \sum_{l} b_{i \ell} p_{\ell}}{\sum_{k} b_{i k} p_{k} \sum_{l} b_{i k} p_{\ell}} \\
\leq & \sum_{j} x_{j} \Delta p_{j}-\sum_{k} x_{k} \Delta p_{k}+\frac{4}{3} \sum_{i} \frac{1}{e_{i}} \sum_{k} x_{i k} \Delta p_{k} \sum_{l} x_{i \ell} \Delta p_{\ell} \quad \text { (by (1) ) } \\
\leq & \frac{4}{3} \sum_{i, j} \frac{x_{i j}}{p_{j}}\left(\Delta p_{j}\right)^{2} \quad(\text { by Claim } 29) \\
= & \frac{4}{3} \sum_{j} \frac{x_{j}}{p_{j}}\left(\Delta p_{j}\right)^{2} \leq 6 \sum_{j} x_{j} \cdot d_{\mathrm{KL}}\left(p_{j}+\Delta p_{j}, p_{j}\right) \quad \text { (by Claim 30). }
\end{aligned}
$$




\section{Complementary CES Utilities}

In this section we consider the weighted update rule,

$$
p_{j}^{t+1}=p_{j}^{t} e^{\left(z_{j} / \gamma_{j}^{t}\right)}
$$

for markets in which every buyer has a complementary CES utility, i.e. the $i$ th buyer has a parameter $\rho_{i}$ in the range $-\infty<\rho_{i}<0$. In addition, the weights $\gamma_{j}^{t}$ are allowed to change from one time step to the next; our updates to price $p_{j}$ will use the weight $\gamma_{j}^{t}=5 \cdot \max \left\{1, x_{j}^{t}\right\}{ }^{17}$ This seems a very natural distributed rule, and indeed a linearization of this rule, $p_{j}^{t+1}=$ $p_{j}^{t}\left[1+\lambda \max \left\{1, z_{j}\right\}\right]^{18}$ was used in the prior works by Cole et al. [12] and Cheung et al. [10].

For these markets we will show that $\phi\left(p^{t}\right)-\phi\left(p^{*}\right)$ reduces by at least a $1-\mu$ factor at each time step, where $0<\mu<1$ depends on the initial price and the market parameters we will specify.

Henceforth, the index $t$ on all the parameters except prices will be implicit.

Notation Recall that $e_{i}$ denotes buyer $i$ 's budget. We set $\gamma=\max _{j} \gamma_{j}$, and again, we let $\Delta p_{j}$ denote $p_{j}^{t+1}-p_{j}^{t}$. We define

$$
c_{i}:=\rho_{i} /\left(\rho_{i}-1\right)
$$

Note that $c_{i}=\sigma_{i}-1$, where $\sigma_{i}=1 /\left(1-\rho_{i}\right)$ is the demand elasticity of the associated CES utility function. Finally, let $c=\max _{i} c_{i}$.

As is well known, the demand for good $j$ when buyer $i$ optimizes her utility is given by

$$
x_{i j}=e_{i} b_{i j} p_{j}^{c_{i}-1} S_{i}^{-1}
$$

where $b_{i j}:=a_{i j}^{1-c_{i}}$ and $S_{i}=\sum_{\ell} b_{i \ell} p_{\ell}^{c_{i}}$. Substituting in (3) shows that this optimal utility equals $e_{i} S_{i}^{-1 / c_{i}}$. It follows that the minimum cost for one unit of utility is $S_{i}^{1 / c_{i}}$. Thus, by Lemma 7 , $\phi$ is given by

$$
\phi\left(p^{t}\right)=\sum_{j} p_{j}^{t}-\sum_{i} e_{i} \log S_{i}^{1 / c_{i}}
$$

In the next two subsections we will show that the potential function in this case satisfies a stronger sandwiching property, as specified in Lemmas 32 and 33 (their proofs occur later in this section). This stronger property immediately yields the claimed bound on the convergence rate (Theorem 35 ).

Claim 31. $\left|p_{j}^{t+1}-p_{j}^{t}\right| \leq \frac{1}{4} p_{j}^{t}$.

Proof. $\left|p_{j}^{t+1}-p_{j}^{t}\right| \leq\left(e^{1 / 5}-1\right) p_{j}^{t} \leq \frac{1}{4} p_{j}^{t}$.

Lemma 32. Suppose that $\left|p_{j}^{t+1}-p_{j}^{t}\right| \leq \frac{1}{4} p_{j}^{t}$ for all $j$. Then

$$
\phi\left(p^{t}\right)-\phi\left(p^{t+1}\right) \geq \frac{1}{2} \sum_{j} \frac{z_{j}^{2} p_{j}^{t}}{\gamma_{j}} .
$$

\footnotetext{
${ }^{17}$ Any greater value for $\gamma_{j}$ would work too, but would entail a proportionate change to the bound in Lemma 33

${ }^{18}$ The $\lambda$ replaces the constant of 5 used here, as a greater range of values for this parameter is needed in markets of substitutes.
} 


\section{Lemma 33.}

$$
\phi\left(p^{t}\right)-\phi\left(p^{*}\right) \leq \max _{j}\left\{10, \frac{5}{2 m_{j}}\right\} \sum_{j} \frac{z_{j}^{2} p_{j}^{t}}{\gamma_{j}},
$$

where $m_{j}=(1-c) / 2$ for $r_{j} \leq 1$ and $m_{j}=\frac{1-r_{j}^{c}+c\left(r_{j}-1\right)}{c\left(r_{j}-1\right)^{2}}$ otherwise, and $r_{j}=p_{j}^{*} / p_{j}^{t}$. Recall that $c=\max _{i} c_{i}$.

It is a simple calculation to check that the definitions of $m_{j}$ coincide at $r_{j}=1$.

The following claim gives a lower bound on $m_{j}$ and is shown in the appendix.

Claim 34. Let $h_{c}\left(r_{j}\right)=m_{j} / c$. Then

i. For $0<c<1, h_{c}(r):=\frac{1-r^{c}+c(r-1)}{(r-1)^{2}}$ is a decreasing function of $r$.

ii. $h_{c}(r) / c$ is a decreasing function of $c$.

iii.

$$
m_{j} \geq \min \left\{\frac{\left(2^{1 / c}-1\right)}{2^{1 / c}\left(r_{j}-1\right)}, \frac{\left(2^{1 / c}-1\right) c^{1 /(1-c)}}{2^{2 / c}}\right\}
$$

We can now deduce our main result.

Theorem 35. For all complementary CES markets, for the sequence of prices $p^{t}$ defined by the update rule (22), for all $t$,

$$
\phi\left(p^{t}\right)-\phi\left(p^{*}\right) \leq\left[(1-\Theta(1)]^{t} d_{K L}\left(p^{*}, p^{0}\right) .\right.
$$

In other words, for any $\epsilon>0, \phi\left(p^{t}\right)-\phi\left(p^{*}\right) \leq \epsilon d_{K L}\left(p^{*}, p^{0}\right)$, if $t=\Omega(\log (1 / \epsilon))$.

Proof.

$$
\begin{aligned}
\phi\left(p^{t+1}\right)-\phi\left(p^{*}\right) & =\phi\left(p^{t}\right)-\phi\left(p^{*}\right)-\left[\phi\left(p^{t}\right)-\phi\left(p^{t+1}\right)\right] \\
& \leq \phi\left(p^{t}\right)-\phi\left(p^{*}\right)-\frac{1}{2} \sum_{j} \frac{z_{j}^{2} p_{j}^{t}}{\gamma_{j}} \quad(\text { by Lemma 32) } \\
& \leq\left[\phi\left(p^{t}\right)-\phi\left(p^{*}\right)\right]\left[1-\frac{1}{2}\left(\max _{j}\left\{10, \frac{5}{2 m_{j}}\right\}\right)^{-1}\right] \quad \text { (by Lemma 33). }
\end{aligned}
$$

Lemma 38, stated in Section 6.3, will show that $r_{j}=p_{j}^{*} / p_{j}$ remains bounded throughout the tatonnement process for all $j$, and hence $m_{j}$ remains bounded away from zero. Consequently,

$$
\phi\left(p^{t}\right)-\phi\left(p^{*}\right)=(1-\Theta(1))\left[\phi\left(p^{t}\right)-\phi\left(p^{*}\right)\right] .
$$




\subsection{The Upper Bound: Good Progress on a Price Update}

The proof of Lemma 32 proceeds in two steps. First, we show that $\phi\left(p^{t+1}\right)-\phi\left(p^{t}\right)+\sum_{j} z_{j}\left[p_{j}^{t+1}-\right.$ $\left.p_{j}^{t}\right] \leq 2 \sum_{j} \frac{x_{j}}{p_{j}}\left[p_{j}^{t+1}-p_{j}^{t}\right]^{2}$. We then choose $\gamma_{j}=5 \cdot \max \left\{1, x_{j}\right\}$. Finally, we deduce the bound in Lemma 32. Our first bound uses the following result.

Lemma 36. Suppose that for all $j,\left|\Delta p_{j}\right| / p_{j} \leq \frac{1}{4}$. Then $\phi(p+\Delta p)-\ell_{\phi}(p+\Delta p ; p) \doteq \phi(p+$ $\Delta p)-\phi(p)+\sum_{j} z_{j} \Delta p_{j} \leq 2 \sum_{j} \frac{x_{j}}{p_{j}}\left(\Delta p_{j}\right)^{2}$.

Proof. As in the proof of Lemma 27, we use two bounds: First, a bound on $\log (1+\epsilon)$, namely:

$$
\log (1+\epsilon) \geq \epsilon-\frac{2}{3} \epsilon^{2}, \text { when }|\epsilon| \leq \frac{7}{24} .
$$

And second, a bound on the following polynomial, which follows from a simple power series expansion: if $\left|\Delta p_{j} / p_{j}\right| \leq 1 / 4$ and $0 \leq c \leq 1$,

$$
\left(p_{j}+\Delta p_{j}\right)^{c} \geq p_{j}^{c}+c p_{j}^{c-1}\left(\Delta p_{j}\right)-\frac{2}{3} c p_{j}^{c-2}\left(\Delta p_{j}\right)^{2} .
$$

We let $D_{\phi}$ denote $\phi(p+\Delta p)-\ell_{\phi}(p+\Delta p ; p)$, for short. Recall that $S_{i}(p)=\sum_{\ell} b_{i \ell} p_{\ell}^{c_{i}}$. Then:

$$
\begin{aligned}
D_{\phi} & =\phi(p+\Delta p)-\phi(p)+\sum_{j} z_{j} \Delta p_{j} \\
& =\sum_{j} \Delta p_{j}+\sum_{j} z_{j} \Delta p_{j}-\sum_{i} \frac{e_{i}}{c_{i}} \log \frac{S_{i}(p+\Delta p)}{S_{i}(p)} . \\
& =\sum_{j} x_{j} \Delta p_{j}-\sum_{i} \frac{e_{i}}{c_{i}} \log \left(\frac{\sum_{\ell} b_{i \ell}\left(p_{\ell}+\Delta p_{\ell}\right)^{c_{i}}}{S_{i}(p)}\right) .
\end{aligned}
$$

As $\rho<0,0<c_{i}<1$. So we can apply (25), yielding:

$$
D_{\phi} \leq \sum_{j} x_{j} \Delta p_{j}-\sum_{i} \frac{e_{i}}{c_{i}} \log \left(1+\frac{\sum_{\ell} b_{i \ell} c_{i} p_{\ell}^{c_{i}-1}\left(\Delta p_{\ell}\right)}{S_{i}(p)}-\frac{\frac{2}{3} \sum_{\ell} b_{i \ell} c_{i} p_{\ell}^{c_{i}-2}\left(\Delta p_{\ell}\right)^{2}}{S_{i}(p)}\right) \text {. }
$$

Recalling from (23) that $x_{i \ell}=e_{i} b_{i \ell} p_{\ell}^{c_{i}-1} / S_{i}(p)$, yields:

$$
D_{\phi} \leq \sum_{j} x_{j} \Delta p_{j}-\sum_{i} \frac{e_{i}}{c_{i}} \log \left(1+\sum_{\ell} c_{i} \frac{x_{i \ell}}{e_{i}}\left(\Delta p_{\ell}\right)-\frac{2}{3} \sum_{\ell} c_{i} \frac{x_{i \ell}}{p_{\ell} e_{i}}\left(\Delta p_{\ell}\right)^{2}\right) .
$$

On applying (24), which we can do as $\sum_{\ell} x_{i \ell} p_{\ell} \leq e_{i}, c_{i} \leq 1$, and $\left|\Delta p_{\ell}\right| / p_{\ell} \leq \frac{1}{4}$, we obtain the bound:

$$
\begin{aligned}
D_{\phi} \leq & \sum_{j} x_{j} \Delta p_{j}-\sum_{i} \frac{e_{i}}{c_{i}}\left(\sum_{\ell} c_{i} \frac{x_{i \ell}}{e_{i}}\left(\Delta p_{\ell}\right)-\frac{2}{3} \sum_{\ell} c_{i} \frac{x_{i \ell}}{p_{\ell} e_{i}}\left(\Delta p_{\ell}\right)^{2}\right) \\
& +\sum_{i} \frac{e_{i}}{c_{i}} \frac{2}{3}\left(\sum_{\ell} c_{i} \frac{x_{i \ell}}{e_{i}}\left(\Delta p_{\ell}\right)-\frac{2}{3} \sum_{\ell} c_{i} \frac{x_{i \ell}}{p_{\ell} e_{i}}\left(\Delta p_{\ell}\right)^{2}\right)^{2} \\
= & \frac{2}{3} \sum_{\ell} \frac{x_{\ell}}{p_{\ell}}\left(\Delta p_{\ell}\right)^{2}+\frac{2}{3} \sum_{i} \frac{c_{i}}{e_{i}}\left(\sum_{\ell} x_{i \ell}\left(\Delta p_{\ell}\right)-\frac{2}{3} \sum_{\ell} \frac{x_{i \ell}}{p_{\ell}}\left(\Delta p_{\ell}\right)^{2}\right)^{2} \\
= & \frac{2}{3} \sum_{\ell} \frac{x_{\ell}}{p_{\ell}}\left(\Delta p_{\ell}\right)^{2}+\frac{2}{3} \sum_{i} \frac{c_{i}}{e_{i}}\left(\sum_{\ell} x_{i \ell}\left(\Delta p_{\ell}\right)\left(1-\frac{2 \Delta p_{\ell}}{3 p_{\ell}}\right)\right)^{2} .
\end{aligned}
$$


Now recall that $\Delta p_{\ell} / p_{\ell} \leq \frac{1}{4}$, to give the bound:

$$
\begin{aligned}
D_{\phi} & \leq \frac{2}{3} \sum_{\ell} \frac{x_{\ell}}{p_{\ell}}\left(\Delta p_{\ell}\right)^{2}+\frac{2}{3} \sum_{i} \frac{c_{i}}{e_{i}}\left(\sum_{\ell} x_{i \ell}\left|\Delta p_{\ell}\right| \cdot \frac{7}{6}\right)^{2} \\
& =\frac{2}{3} \sum_{\ell} \frac{x_{\ell}}{p_{\ell}}\left(\Delta p_{\ell}\right)^{2}+\frac{49}{54} \sum_{i} \frac{1}{e_{i}}\left(\sum_{\ell} x_{i \ell}\left|\Delta p_{\ell}\right|\right)^{2} \quad\left(\text { as } c_{i} \leq 1\right) \\
& =\frac{2}{3} \sum_{\ell} \frac{x_{\ell}}{p_{\ell}}\left(\Delta p_{\ell}\right)^{2}+\frac{49}{54} \sum_{i} \frac{1}{e_{i}} \sum_{j, k} x_{i j} x_{i k}\left|\Delta p_{j}\right|\left|\Delta p_{k}\right| \\
& \leq\left(\frac{2}{3}+\frac{49}{54}\right) \sum \frac{x_{\ell}}{p_{\ell}}\left(\Delta p_{\ell}\right)^{2} \quad \text { (by Claim 29) } \\
& \leq 2 \sum \frac{x_{\ell}}{p_{\ell}}\left(\Delta p_{\ell}\right)^{2} .
\end{aligned}
$$

Proof of Lemma 32. Recall that $\Delta p_{j}=p_{j}^{t+1}-p_{j}^{t}$ and that $p_{j}^{t+1}=p_{j}^{t} e^{\left(z_{j} / \gamma_{j}\right)}$. By Lemma 36 .

$$
\phi\left(p^{t}\right)-\phi\left(p^{t+1}\right) \geq \sum_{j} z_{j}\left[p_{j}^{t+1}-p_{j}^{t}\right]-2 \sum_{j} \frac{x_{j}}{p_{j}^{t}}\left[p_{j}^{t+1}-p_{j}^{t}\right]^{2}
$$

Next, using the formula for $p^{t+1}$ and the fact that $\gamma_{j} \geq 5 x_{j}$ gives the bound:

$$
\begin{aligned}
\phi\left(p^{t}\right)-\phi\left(p^{t+1}\right) & \geq \sum_{j} z_{j} p_{j}^{t}\left[e^{\left(z_{j} / \gamma_{j}\right)}-1\right]-\frac{2}{5} \sum_{j} \gamma_{j} p_{j}^{t}\left[e^{\left(z_{j} / \gamma_{j}\right)}-1\right]^{2} \\
& =\sum_{j} z_{j} p_{j}^{t}\left[e^{\left(z_{j} / \gamma_{j}\right)}-1\right]\left(1-\frac{2}{5} \frac{\gamma_{j}}{z_{j}}\left[e^{\left(z_{j} / \gamma_{j}\right)}-1\right]\right) \\
& \geq \sum_{z_{j} \geq 0} \frac{z_{j}^{2} p_{j}^{t}}{\gamma_{j}}\left(1-\frac{2}{5} \cdot \frac{10}{9}\right)+\sum_{z_{j}<0} \frac{z_{j}^{2} p_{j}^{t}}{\gamma_{j}} \frac{9}{10}\left(1-\frac{2}{5}\right) \\
& \geq \frac{1}{2} \sum_{j} \frac{z_{j}^{2} p_{j}^{t}}{\gamma_{j}} .
\end{aligned}
$$

\subsection{An Upper Bound on the Distance to Equilibrium}

Lemma 37. Suppose that $p_{j}^{*} / p_{j} \leq r_{j}$ for all $j$, where $r_{j} \geq 1$. Let $c=\max _{i} c_{i}$. Then

$$
\phi\left(p^{*}\right)-\ell_{\phi}\left(p^{*} ; p\right) \geq \sum_{\ell} \frac{h_{c}\left(r_{\ell}\right)}{c} x_{\ell} \cdot \frac{\left(p_{\ell}^{*}-p_{\ell}\right)^{2}}{p_{\ell}} \text {. }
$$

Proof. As with previous lemmas, we use a bound on the polynomial $\left(p_{j}^{*}-p_{j}\right)^{c_{i}}$, but now we use the bound given by Claim 34 i. Specifically, if $p_{j}^{*} / p_{j} \leq r_{j}$ and $0<c \leq 1, h_{c}\left(p_{j}^{*} / p_{j}\right) \geq h_{c}\left(r_{j}\right)$, i.e.

$$
\frac{\frac{1}{p_{j}^{c}}\left[p_{j}^{c}-\left(p_{j}^{*}\right)^{c}+c p_{j}^{c-1}\left(p_{j}^{*}-p_{j}\right)\right]}{\frac{1}{p_{j}^{2}}\left(p_{j}^{*}-p_{j}\right)^{2}} \geq h_{c}\left(r_{j}\right),
$$


SO

$$
\left(p_{j}^{*}\right)^{c} \leq p_{j}^{c}+c p_{j}^{c-1}\left(p_{j}^{*}-p_{j}\right)-h_{c}\left(r_{j}\right) p_{j}^{c-2}\left(p_{j}^{*}-p_{j}\right)^{2} .
$$

We also use a simple bound on the $\log$ function, namely $\log (1+\epsilon) \leq \epsilon$ for $\epsilon \geq-1$. To avoid clutter, we omit the superscript $t$ on the prices.

Let $\Delta^{*} p_{j}=p_{j}^{*}-p_{j}$. Then

$$
\phi\left(p^{*}\right)-\ell_{\phi}\left(p^{*} ; p\right)=\sum_{j} x_{j} \Delta^{*} p_{j}-\sum_{i} \frac{e_{i}}{c_{i}} \log \left(\frac{\sum_{\ell} b_{i \ell}\left(p_{l}^{*}\right)^{c_{i}}}{S_{i}(p)}\right) .
$$

Recalling that $S_{i}(p)=\sum_{l} b_{i \ell}\left(p_{\ell}\right)^{c_{i}}$ and using the upper bound on $\left(p_{j}^{*}\right)^{c_{i}}$ from (28) gives:

$$
\begin{aligned}
& \phi\left(p^{*}\right)-\ell_{\phi}\left(p^{*} ; p\right) \\
\geq & \sum_{j} x_{j} \Delta^{*} p_{j}-\sum_{i} \frac{e_{i}}{c_{i}} \log \left(1+\frac{\sum_{\ell} b_{i \ell} c_{i} p_{\ell}^{c_{i}-1}\left(\Delta^{*} p_{\ell}\right)}{S_{i}(p)}-\frac{\sum_{\ell} b_{i \ell} h_{c_{i}}\left(r_{\ell}\right) p_{\ell}^{c_{i}-2}\left(\Delta^{*} p_{\ell}\right)^{2}}{S_{i}(p)}\right) \\
= & \sum_{j} x_{j} \Delta^{*} p_{j}-\sum_{i} \frac{e_{i}}{c_{i}} \log \left(1+\sum_{\ell} c_{i} \frac{x_{i \ell}}{e_{i}}\left(\Delta^{*} p_{\ell}\right)-\sum_{\ell} h_{c_{i}}\left(r_{\ell}\right) \frac{x_{i \ell}}{p_{\ell} e_{i}}\left(\Delta^{*} p_{\ell}\right)^{2}\right) .
\end{aligned}
$$

On noting that the argument for the log is positive (as it is an upper bound for $S_{i}\left(p^{*}\right) / S_{i}(p)$ ), we can apply the bound $\epsilon \geq \log (1+\epsilon)$ for $\epsilon \geq-1$ to give:

$$
\begin{aligned}
\phi\left(p^{*}\right)-\ell_{\phi}\left(p^{*} ; p\right) & \geq \sum_{j} x_{j} \Delta^{*} p_{j}-\sum_{i} \frac{e_{i}}{c_{i}}\left(\sum_{\ell} c_{i} \frac{x_{i \ell}}{e_{i}}\left(\Delta^{*} p_{\ell}\right)-\sum_{\ell} h_{c_{i}}\left(r_{\ell}\right) \frac{x_{i \ell}}{p_{\ell} e_{i}}\left(\Delta^{*} p_{\ell}\right)^{2}\right) \\
& =\sum_{i} \sum_{\ell} \frac{h_{c_{i}}\left(r_{\ell}\right)}{c_{i}} x_{i \ell} \frac{\left(\Delta^{*} p_{\ell}\right)^{2}}{p_{\ell}} \\
& \geq \sum_{i} \sum_{\ell} \frac{h_{c}\left(r_{\ell}\right)}{c} x_{i \ell} \frac{\left(\Delta^{*} p_{\ell}\right)^{2}}{p_{\ell}} \quad \text { (by Claim 34 ii.) } \\
& =\sum_{\ell} \frac{h_{c}\left(r_{\ell}\right)}{c} x_{\ell} \frac{\left(\Delta^{*} p_{\ell}\right)^{2}}{p_{\ell}} .
\end{aligned}
$$

Proof of Lemma 33. Note that $m_{j}=h_{c}\left(r_{j}\right) / c$. Then, by Lemma 37 .

$$
\begin{aligned}
\phi\left(p^{t}\right)-\phi\left(p^{*}\right) & =l_{\phi}\left(p^{*}, p^{t}\right)-\phi\left(p^{*}\right)-\nabla \phi\left(p^{t}\right) \cdot\left(p^{*}-p^{t}\right) \\
& \leq \sum_{j} z_{j}\left(p_{j}^{*}-p_{j}^{t}\right)-\sum_{j} m_{j} x_{j} \frac{\left(p_{j}^{*}-p_{j}^{t}\right)^{2}}{p_{j}^{t}} \\
& \leq \max _{p^{\prime}} \sum_{j}\left(z_{j}\left(p_{j}^{\prime}-p_{j}^{t}\right)-m_{j} x_{j} \frac{\left(p_{j}^{\prime}-p_{j}^{t}\right)^{2}}{p_{j}^{t}}\right) .
\end{aligned}
$$

There are two cases.

Case 1: $0 \leq x_{j} \leq 1 / 2$.

Then $-1 \leq z_{j} \leq-1 / 2$ and hence $z_{j} \geq-2 z_{j}^{2}$. Thus 


$$
z_{j}\left(p_{j}^{\prime}-p_{j}^{t}\right)-m_{j} x_{j} \frac{\left(p_{j}^{\prime}-p_{j}^{t}\right)^{2}}{p_{j}^{t}} \leq-z_{j} p_{j}^{t} \leq 2 z_{j}^{2} p_{j}^{t}=2 \gamma_{j} \frac{z_{j}^{2} p_{j}^{t}}{\gamma_{j}}
$$

As $x_{j} \leq 1 / 2<1,2 \gamma_{j}=10$. Hence

$$
z_{j}\left(p_{j}^{\prime}-p_{j}^{t}\right)-m_{j} x_{j} \frac{\left(p_{j}^{\prime}-p_{j}^{t}\right)^{2}}{p_{j}^{t}} \leq 10 \frac{z_{j}^{2} p_{j}^{t}}{\gamma_{j}}
$$

Case 2: $x_{j} \geq 1 / 2$.

$z_{j}\left(p_{j}^{\prime}-p_{j}^{t}\right)-m_{j} x_{j} \frac{\left(p_{j}^{\prime}-p_{j}^{t}\right)^{2}}{p_{j}^{t}}$ is a quadratic function of $\left(p_{j}^{\prime}-p_{j}^{t}\right)$. The quadratic function is maximized when $\left(p_{j}^{\prime}-p_{j}^{t}\right)=\frac{z_{j} p_{j}^{t}}{2 m_{j} x_{j}}$, with its maximum value being $\frac{z_{j}^{2} p_{j}^{t}}{4 m_{j} x_{j}}=\frac{\gamma_{j}}{4 m_{j} x_{j}} \frac{z_{j}^{2} p_{j}^{t}}{\gamma_{j}}$.

As $x_{j} \geq 1 / 2$ and $\gamma_{j}=5 \cdot \max \left\{1, x_{j}\right\}, \gamma_{j} / x_{j} \leq 10$. Hence

$$
z_{j}\left(p_{j}^{\prime}-p_{j}^{t}\right)-m_{j} x_{j} \frac{\left(p_{j}^{\prime}-p_{j}^{t}\right)^{2}}{p_{j}^{t}} \leq \frac{5}{2 m_{j}} \frac{z_{j}^{2} p_{j}^{t}}{\gamma_{j}}
$$

Combining the two cases yields the result.

\subsection{Bounding $m_{j}$}

Let $p_{\mathrm{U}}=\max _{j}\left\{p_{j}^{\circ}\right\}$, the maximum initial price, $U=\max \left\{p_{\mathrm{U}}, M\right\}$, and $L^{*}=\min _{j}\left\{p_{j}^{*}\right\}$. The following bound is shown in the appendix.

Lemma 38. Let $\bar{U}=U$ for any continuous tatonnement, and let $\bar{U}=2 U$ for the discrete tatonnement with update rule 22]. For any continuous tatonnement, $p_{j}^{*} / p_{j}^{t} \leq \max \left\{p_{j}^{*} / p_{j}^{\circ},\left(L^{*} / \bar{U}\right)^{\min _{i} \rho_{i}}\right\}$, and for the discrete tatonnement,$p_{j}^{*} / p_{j}^{t} \leq 2 \cdot \max \left\{p_{j}^{*} / p_{j}^{\circ},\left(L^{*} / \bar{U}\right)^{\min _{i} \rho_{i}}\right\}$.

\section{Substitute CES Utilities}

The analysis in Section 6 can be extended to Fisher markets with substitute CES utilities, i.e. CES utility functions with parameter $\rho \geq 0$. Cole and Fleischer [12] showed that tatonnement converges in these markets via a different potential function. For completeness, we reprove this result here with the technique developed in Section 6. We will prove lemmas similar to Lemmas 32, 33, 36, 37 and then prove a theorem similar to Theorem 35 .

For substitute CES utilities, the parameter $c_{i}=\rho_{i} /\left(\rho_{i}-1\right)$ is negative, while it is positive in the complementary case. Due to the sign switch, some of the proofs of the lemmas in this section differ from the corresponding proofs in Section 6 .

\subsection{The Upper Bound: Good Progress on a Price Update}

In this section, we defer most of the proofs to the appendix, due to their similarility with the proofs in the preceding section.

Let $c_{\min }=\min _{i} c_{i}$. 
Lemma 39. Suppose that for all $j,\left|\Delta p_{j}\right| / p_{j} \leq \min \left\{1 / 4,1 /\left|c_{\min }\right|\right\}$. Then $\phi(p+\Delta p)-\ell_{\phi}(p+$ $\Delta p ; p) \leq\left(1-c_{\min }\right) \sum_{j} \frac{x_{j}}{p_{j}}\left(\Delta p_{j}\right)^{2}$.

To allow us to apply Lemma 39 in our analysis, we will require that $\gamma_{j}^{t}=5 \cdot \max \{1,(1-$ $\left.\left.c_{\min }\right) / 2\right\} \cdot \max \left\{1, x_{j}^{t}\right\}$.

Lemma 40. Suppose that $\left|p_{j}^{t+1}-p_{j}^{t}\right| \leq \min \left\{1 / 4,1 /\left|c_{\min }\right|\right\} \cdot p_{j}^{t}$ for all $j$. Then

$$
\phi\left(p^{t}\right)-\phi\left(p^{t+1}\right) \geq \frac{1}{2} \sum_{j} \frac{z_{j}^{2} p_{j}^{t}}{\gamma_{j}}
$$

Proof. It is almost identical to the proof of Lemma 32. It uses the bound from Lemma 39 instead of the bound from Lemma 36. This changes the factor 2 in $(26)$ to $1-c_{\min }$, and then the new value for $\gamma_{j}$ yields Equation (27). The rest of the proof is identical.

\subsection{An Upper Bound on the Distance to Equilibrium}

Lemma 41. Suppose that $p_{j}^{*} / p_{j} \leq r_{j}$ for all $j$, where $r_{j} \geq 1$. Then

$$
\phi\left(p^{*}\right)-\ell_{\phi}\left(p^{*} ; p\right) \geq \sum_{j} \frac{r_{j}-1-\log r_{j}}{\left(r_{j}-1\right)^{2}} x_{j} \frac{\left(p_{j}^{*}-p_{j}\right)^{2}}{p_{j}} .
$$

Lemma 42.

$$
\phi\left(p^{t}\right)-\phi\left(p^{*}\right) \leq \max \left\{1,\left(1-c_{\min }\right) / 2\right\} \sum_{j} \max _{j}\left\{10, \frac{5\left(r_{j}-1\right)^{2}}{2\left(r_{j}-1-\log r_{j}\right)}\right\} \cdot \frac{z_{j}^{2} p_{j}^{t}}{\gamma_{j}},
$$

where $r_{j}=\max \left\{1, p_{j}^{*} / p_{j}\right\}$.

Proof. The proof is almost identical to the proof of Lemma 33. The ratio $\left(r_{j}-1-\log r_{j}\right) /\left(\left(r_{j}-\right.\right.$ $1)^{2}$ ) from Lemma 41 plays the role of $h_{c}\left(r_{j}\right) / c=m_{j}$ from Lemma 37. In the proof of Lemma 33 , we used the fact that $\gamma_{j}=5$ in Case 1 , and that $\gamma_{j}=5 x_{j}$ in Case 2. Here, we replace the 5 with $5 \cdot \max \left\{1,\left(1-c_{\min }\right) / 2\right\}$, yielding the new bound.

Theorem 43. For all substitute CES markets, for the sequence of prices $p^{t}$ defined by update rule 22 , for all $t$,

$$
\phi\left(p^{t}\right)-\phi\left(p^{*}\right) \leq\left[(1-\Theta(1)]^{t} d_{K L}\left(p^{*}, p^{0}\right)\right.
$$

In other words, for any $\epsilon>0, \phi\left(p^{t}\right)-\phi\left(p^{*}\right) \leq \epsilon d_{K L}\left(p^{*}, p^{0}\right)$, if $t=\Omega(\log (1 / \epsilon))$.

Proof. This is almost identical to the proof of Theorem 35. Instead of the bounds from Lemmas 32 and 33 , we use the bounds from Lemmas 40 and 42 . This gives

$$
\phi\left(p^{t+1}\right)-\phi\left(p^{*}\right) \leq \frac{\phi\left(p^{t}\right)-\phi\left(p^{*}\right)}{\max \left\{1,\left(1-c_{\min }\right) / 2\right\}}\left[1-\frac{1}{2}\left(\max _{j}\left\{10, \frac{2\left(r_{j}-1-\log r_{j}\right)}{\left(r_{j}-1\right)^{2}}\right\}\right)^{-1}\right] .
$$

We then note that Cole and Fleisher [12] showed that $\max _{j} r_{j}$ remains bounded by its initial valure $r_{j}^{0}$ throughout the tatonnement process, yielding the desired bound (we consider $r_{j}^{0}$ to be a constant). 


\section{Discussion}

We have shown that discrete versions of tatonnement converge for Leontief and CES utilities. The main open question is whether these convergence results extend to the Ongoing Market model defined by Cole and Fleisher [12]. In this model, the market repeats from one time period to the next, and excess demands and supplies are carried forward to successive time periods using finite buffers, which they called warehouses. The purpose of this model was to provide a more natural setting for the tatonnement update process.

There are two aspects to the Ongoing Market that our results do not address.

- Warehouses. There is a separate warehouse for each good. The price update for each good is adjusted to take account of whether the warehouse is relatively full or empty. The goal is to show, as in [10], that the tatonnement price update converges to the equilibrium prices and that in addition this can be achieved without having the warehouse either overflow or run out of stock, and further that it too converges to an ideal state, namely half-full. We conjecture that this is possible for the markets with CES utilities at least.

- Asynchrony. This allows the prices to be updated independently, at separate times, with the sole constraint that each price updates at least once per time unit. Further, each price update uses the accumulated demand since the previous update, as opposed to the instantaneous demand, to determine its size. Again, both the asynchrony itself, and the price update rule, are intended to provide a process that seems more natural.

We also conjecture that this variant of the price update will converge for markets with CES utilities.

The previous analyses for the Ongoing Market used non-trivial amortized arguments. It seems they will not extend to the present setting, for they were intrinsically linear, whereas the potential function employed here for the CES utilities is quadratic. Still, we suspect there may be extensions of the current analyses that will lead to the conjectured results.

Also, it would be interesting to resolve the behavior of a discrete tatonnement for nested CES Fisher markets. Our belief is that this will require resolving whether this class of utilities is controlled.

\section{Acknowledgments}

Yun Kuen Cheung and Richard Cole were supported in part by NSF grant CCF-1217989.

We thank Lin Xiao for his pointers to the literature on strong convexity and gradient descent. The first author thanks Po Lam Yung for useful discussions on Section 4 .

\section{References}

[1] K. Arrow, H. Block, and L. Hurwicz. On the stability of the competitive equilibrium: II. Econometrica, 27(1):82-109, 1959.

[2] K. J. Arrow and G. Debreu. Existence of an equilibrium for a competitive economy. Econometrica, 22(3):265-290, 1954. 
[3] Claude Berge. Topological Spaces. Oliver \& Boyd, Edinburgh, London, 1963.

[4] Benjamin Birnbaum, Nikhil Devanur, and Lin Xiao. Distributed algorithms via gradient descent for fisher markets. In ACM Conference on Electronic Commerce, EC'11, pages 127-136, 2011.

[5] Stephen Boyd and Lieven Vandenberghe. Convex Optimization. Cambridge University Press, 2004.

[6] William C. Brainard and Herbert E. Scarf. How to compute equilibrium prices in 1891. American Journal of Economics and Sociology, 64(1):57-83, 2005.

[7] G. Chen and M. Teboulle. Convergence analysis of a proximal-like minimization algorithm using Bregman functions. SIAM Journal on Optimization, 3(3):538-543, August 1993.

[8] Ning Chen, Xiaotie Deng, Hongyang Zhang, and Jie Zhang. Incentive ratios of fisher markets. In Proceedings of the 39th international colloquium conference on Automata, Languages, and Programming - Volume Part II, ICALP'12, pages 464-475, Berlin, Heidelberg, 2012. Springer-Verlag.

[9] Ning Chen, Xiaotie Deng, and Jie Zhang. How profitable are strategic behaviors in a market? In Proceedings of the 19th European conference on Algorithms, ESA'11, pages 106-118, Berlin, Heidelberg, 2011. Springer-Verlag.

[10] Yun Kuen Cheung, Richard Cole, and Ashish Rastogi. Tatonnement in ongoing markets of complementary goods. In ACM Conference on Electronic Commerce, EC'12, pages 337-354, 2012.

[11] Bruno Codenotti, Benton Mccune, and Kasturi Varadarajan. Market equilibrium via the excess demand function. In Proceedings of the Thirty Seventh Annual ACM Symposium on Theory of Computing, STOC'05, pages 74-83, 2005.

[12] Richard Cole and Lisa Fleischer. Fast-converging tatonnement algorithms for one-time and ongoing market problems. In Proceedings of the Fortieth Annual ACM Symposium on Theory of Computing, STOC'08, pages 315-324. ACM Press, 2008.

[13] Nikhil R Devanur. Fisher markets and convex programs. Working paper available from http://research.microsoft.com/en-us/um/people/ nikdev/, 2009.

[14] E. Eisenberg. Aggregation of utility functions. Management Sciences, 7(4):337-350, 1961.

[15] Edmund Eisenberg and David Gale. Consensus of subjective probabilities: The parimutuel method. Ann. Math. Statist., 30(1):165-168, 1959.

[16] Franklin M. Fisher. On price adjustment without an auctioneer. The Review of Economic Studies, 39(1):pp. 1-15, 1972.

[17] Lisa Fleischer, Rahul Garg, Sanjiv Kapoor, Rohit Khandekar, and Amin Saberi. A fast and simple algorithm for computing market equilibria. In WINE, pages 19-30, 2008.

[18] George Green. An Essay on the Application of Mathematical Analysis to the Theories of Electricity and Magnetism. Nottingham, England: T. Wheelhouse, 1828. 
[19] Masayoshi Hirota, Ming Hsu, Charles R. Plott, and Brian W. Rogers. Divergence, closed cycles and convergence in Scarf environments: Experiments in the dynamics of general equilibrium systems. Working Papers 1239, California Institute of Technology, Division of the Humanities and Social Sciences, October 2005.

[20] Kamal Jain and Vijay V. Vazirani. Eisenberg-Gale markets: algorithms and gametheoretic properties. Games and Economic Behavior, 70(1), 2010.

[21] Kamal Jain, Vijay V. Vazirani, and Yinyu Ye. Market equilibria for homothetic, quasiconcave utilities and economies of scale in production. In Proceedings of the Sixteenth Annual ACM-SIAM Symposium on Discrete Algorithms, SODA'05, pages 63-71, 2005.

[22] Wouter J. Keller. A Nested CES-Type Utility Function and Its Demand and Price-Index Functions. European Economic Review, 7(2):175-186, 1976.

[23] F. P. Kelly and V. V. Vazirani. Rate control as a market equilibrium. Manuscript, 2002.

[24] Andreu Mas-Collel, Michael D. Whinston, and Jerry R. Green. Microeconomic Theory. Oxford University Press, 1995.

[25] Bernard Riemann. Grundlagen füreinen allgemeine Theorie der Functionen einer veränderlichen complexen Grösse (Basis for a general theory of functions of a variable complex quantity). Göttingen, (Germany): Adalbert Rente, 1867.

[26] Donald John Roberts and Andrew Postlewaite. The incentives for price-taking behavior in large exchange economies. Econometrica, 44(1):115-27, January 1976.

[27] H. Scarf. Some examples of global instability of the competitive equilibrium. International Econ. Review, 1:157-172, 1960.

[28] G.V. Smirnov. Introduction to the Theory of Differential Inclusions. Crm Proceedings \& Lecture Notes. American Mathematical Society, 2002.

[29] Hirofumi Uzawa. Walras' tatonnement in the theory of exchange. Review of Economic Studies, 27:182-94, 1960.

[30] Léon Walras. Eléments d'Economie Politique Pure. Corbaz, Lausanne, 1874. (Translated as: Elements of Pure Economics. Homewood, IL: Irwin, 1954.). 


\section{A Additional Proofs}

\section{A.1 Proofs for Continuous Time Tatonnement}

Proof of Lemma 20. We first observe that in Fisher markets prices remain bounded. The following notation will be helpful. Let $U$ be the maximum initial price and $M$ the total money in the market, and let $\bar{U}=\max \{U, M\}$. Observe that for any $j$, if $p_{j}=\bar{U}$, then $x_{j} \leq 1$, and consequently any tatonnement rule will not increase $p_{j}$ beyond $\bar{U}$.

We can now show that for Fisher markets $1 / h^{\prime \prime}$ remains bounded. For $h^{\prime \prime}>0$ and consequently in the bounded region $\mathbb{R}_{+}^{n} \cap\{p \leq \bar{U} \mathbb{1}\}$ the supremum of $1 / h^{\prime \prime}$ is its maximum, which is therefore finite.

Thus to prove the result of the lemma it suffices to show that $-\nabla_{j} \phi(p)=z_{j}(p)$ remains bounded throughout the tatonnement.

We begin by considering substitutes CES utilities. Let $f=\min _{j}\left\{p_{j} / p_{j}^{*}, 1\right\}$. Cole and Fleischer [12] showed that if $p_{j}=f p_{j}^{*}$, then $x_{j} \geq 1$. Thus if $p_{j}$ is ever reduced to $f p_{j}^{*}$, the tatonnement update will not decrease it further. Consequently, for all $j, p_{j} \geq f p_{j}^{*}$ throughout the tatonnement process. Hence $x_{j} \leq M /\left(f p_{j}^{*}\right)$ throughout the tatonnement process, for all $j$, where $M$ is the total money in the market. It follows that $z_{j} \leq M /\left(f p_{j}^{*}\right)-1$, for all $j$. This analysis applies to linear utilities too.

We turn to complementary CES utilities. By Lemma $38, p_{j}^{t} \geq p_{j}^{*} \cdot \min \left\{p_{j}^{0} / p_{j}^{*},\left(\bar{U} / L^{*}\right)^{\min _{i} \rho_{i}}\right\}$, where $L^{*}=\min _{j}\left\{p_{j}^{*}\right\}$. It follows that the demands are upper bounded by

$x_{j} \leq \max \left\{p_{j}^{*} / p_{j}^{0},\left(L^{*} / \bar{U}\right)^{\min _{i} \rho_{i}}\right\}$, and hence $z_{j} \leq \max \left\{p_{j}^{*} / p_{j}^{0},\left(L^{*} / \bar{U}\right)^{\min _{i} \rho_{i}}\right\}-1$.

Finally, we consider Leontief utilities. By Lemma $28, x_{j}^{t} \leq x_{j}^{\circ}+\sum_{i} \max _{k} \frac{b_{i j}}{b_{i k}}$, and hence $z_{j}^{t} \leq z_{j}^{\circ}+\sum_{i} \max _{k} \frac{b_{i j}}{b_{i k}}$.

\section{A.2 Proofs for Complementary CES}

Proof of Claim 29. This result follows by rewriting $e_{i}$ as $\sum_{k} x_{i k} p_{k}$.

$$
\begin{aligned}
e_{i} \sum_{l} \frac{x_{i l}}{p_{\ell}}\left(\Delta p_{\ell}\right)^{2} & =\sum_{l} \frac{x_{i l}\left(\sum_{k} x_{i k} p_{k}\right)}{p_{\ell}}\left(\Delta p_{\ell}\right)^{2}=\sum_{l, k} x_{i l} x_{i k} \frac{p_{k}}{p_{\ell}}\left(\Delta p_{\ell}\right)^{2} \\
& =\sum_{l} x_{i l}^{2}\left(\Delta p_{\ell}\right)^{2}+\sum_{k, l: k \neq l} x_{i k} x_{i l} \frac{p_{k}}{p_{\ell}}\left(\Delta p_{\ell}\right)^{2} \\
& =\sum_{l} x_{i l}^{2}\left(\Delta p_{\ell}\right)^{2}+\sum_{k<l} x_{i k} x_{i l}\left(\frac{p_{k}}{p_{\ell}}\left(\Delta p_{\ell}\right)^{2}+\frac{p_{\ell}}{p_{k}}\left(\Delta p_{k}\right)^{2}\right) .
\end{aligned}
$$

Now, we apply the AM-GM inequality:

$$
\begin{aligned}
e_{i} \sum_{l} \frac{x_{i l}}{p_{\ell}}\left(\Delta p_{\ell}\right)^{2} & \geq \sum_{l} x_{i l}^{2}\left(\Delta p_{\ell}\right)^{2}+\sum_{k<l} x_{i k} x_{i l} \cdot 2\left|\Delta p_{\ell} \| \Delta p_{k}\right| \\
& =\sum_{j, k} x_{i j} x_{i k}\left|\Delta p_{j}\right|\left|\Delta p_{k}\right| .
\end{aligned}
$$


Proof of Claim 30. We use the bound $\log x \geq x-\frac{11}{18} x^{2}$ for $|x| \leq \frac{1}{4}$.

$$
d_{\mathrm{KL}}\left(p_{j}+\Delta p_{j}, p_{j}\right)=\left(p_{j}+\Delta p_{j}\right) \log \left(p_{j}+\Delta p_{j}\right)-\left(p_{j}+\Delta p_{j}\right)-p_{j} \log p_{j}+p_{j}-\left(\log p_{j}\right) \Delta p_{j}
$$

(by (4) and (5))

$$
\begin{aligned}
& =-\Delta p_{j}+\left(p_{j}+\Delta p_{j}\right) \log \left(1+\frac{\Delta p_{j}}{p_{j}}\right) \\
& \geq-\Delta p_{j}+\left(p_{j}+\Delta p_{j}\right)\left(\frac{\Delta p_{j}}{p_{j}}-\frac{11}{18} \frac{\left(\Delta p_{j}\right)^{2}}{p_{j}^{2}}\right) \\
& =\frac{7}{18} \frac{\left(\Delta p_{j}\right)^{2}}{p_{j}}\left(1-\frac{11}{7} \frac{\Delta p_{j}}{p_{j}}\right)=\frac{7}{18} \frac{17}{28} \frac{\left(\Delta p_{j}\right)^{2}}{p_{j}} \\
& \geq \frac{2}{9} \frac{\left(\Delta p_{j}\right)^{2}}{p_{j}} .
\end{aligned}
$$

(by 4 and

Proof of Claim 34. (i) and (ii) are readily checked by calculus. For (iii) we argue as follows. For $r_{j} \geq 2$,

$$
\begin{aligned}
m_{j} & =\frac{1-\left(r_{j}-1\right)^{c}\left[1+1 /\left(r_{j}-1\right)\right]^{c}+c\left(r_{j}-1\right)}{c\left(r_{j}-1\right)^{2}} \\
& \geq \frac{1-\left(r_{j}-1\right)^{c}\left[1+c /\left(r_{j}-1\right)-\frac{1}{2} c(1-c) /\left(r_{j}-1\right)^{2}\right]+c\left(r_{j}-1\right)}{c\left(r_{j}-1\right)^{2}} \quad\left(\text { as } r_{j} \geq 2\right) \\
& \geq \frac{\left.c\left(r_{j}-1\right)+1-\left(r_{j}-1\right)^{c}-c /\left(r_{j}-1\right)-\frac{1}{2} c(1-c) /\left(r_{j}-1\right)^{2}\right)^{1-c}}{c\left(r_{j}-1\right)^{2}} \\
& \geq \frac{c\left(r_{j}-1\right)-\left(r_{j}-1\right)^{c}}{c\left(r_{j}-1\right)^{2}} \quad\left(\text { as } 1 \geq c\left[1+\frac{1}{2}(1-c)\right], \text { for } c \leq 1\right) .
\end{aligned}
$$

If $r_{j}-1=c^{-1 /(1-c)}, c\left(r_{j}-1\right)=\left(r_{j}-1\right)^{c}$. So when $r_{j}-1=2^{1 / c} c^{-1 /(1-c)}$,

$$
c\left(r_{j}-1\right)-\left(r_{j}-1\right)^{c}=\left(2^{1 / c}-1\right) c \cdot c^{-1 /(1-c)} .
$$

And as $c\left(r_{j}-1\right)$ grows faster than $\left(r_{j}-1\right)^{c}$, for $r_{j}-1 \geq 2^{1 / c} c^{-1 /(1-c)}$,

$$
c\left(r_{j}-1\right)-\left(r_{j}-1\right)^{c} \geq\left(2^{1 / c}-1\right) c\left(r_{j}-1\right) 2^{-1 / c} .
$$

Then $m_{j} \geq\left(2^{1 / c}-1\right) 2^{-1 / c} /\left(r_{j}-1\right)$.

$m_{j}$ is a decreasing function of $r_{j}$. It follows that for $0 \leq r_{j}-1 \leq 2^{1 / c} c^{-1 /(1-c)}$,

$$
m_{j} \geq\left(2^{1 / c}-1\right) 2^{-2 / c} c^{1 /(1-c)} .
$$

Proof of Lemma 38. We first not two observations.

Observation 1. No price will exceed $\bar{U}$ during the entire tatonnement.

Reason. Suppose not, then let $t=\tau$ be the first time when some price, say $p_{k}$, exceed $\bar{U}$. Then $p_{k}^{\tau} \geq M$ and $x_{k}^{\tau} \leq M / p_{k}^{\tau} \leq 1$. In the continuous tatonnement, the price update rule will not increase $p_{k}$ any further. 
For the discrete tatonnement we argue as follows. At $t=\tau-1, p_{k}^{\tau-1}<\bar{U}=2 U$. But $p_{k}^{\tau-1} \geq U \geq M$, as $p_{k}$ can at most double in one time unit. By the same argument as for $x_{k}^{\tau}$, $x_{k}^{\tau-1} \leq 1$. By the price update rule, $p_{k}^{\tau} \leq p_{k}^{\tau-1}<2 U$, a contradiction.

Observation 2. $p_{k} \geq \min \left\{p_{k}^{\circ},\left(\bar{U} / L^{*}\right)^{\min _{i} \rho_{i}} p_{k}^{*}\right\}$ throughout the entire continuous tatonnement process, and half this value in the discrete case.

Reason. Suppose that for some $k, p_{k} \leq L^{*}\left(\bar{U} / L^{*}\right)^{\min _{i} \rho_{i}} p_{k}^{*}$. We claim that $x_{k} \geq 1$. At equilibrium prices, all demands equal 1 . If the prices are all raised by a factor of $\frac{\bar{U}}{L^{*}}$, then all demands equal $\frac{L^{*}}{\bar{U}}$. Note that now all prices are at least $\bar{U}$.

Now reduce the price of $p_{k}$ from $\frac{\bar{U}}{L^{*}} p_{k}^{*}$ to $\left(\frac{\bar{U}}{L^{*}}\right)^{\min _{i} \rho_{i}} p_{k}^{*}$, that is, reduce the price by a factor of $\left(\frac{\bar{U}}{L^{*}}\right)^{1-\min _{i} \rho_{i}}$. The price reduction can only decrease $S_{i}$. It then follows from 23 that the new demand $x_{k}^{\prime}$ for good $k$ is bounded as follows

$$
x_{k}^{\prime} \geq x_{k} \cdot\left[\left(\frac{\bar{U}}{L^{*}}\right)^{1-\min _{i} \rho_{i}}\right]^{1 /\left(1-\min _{i} \rho_{i}\right)}=\frac{L^{*}}{\bar{U}} \cdot \frac{\bar{U}}{L^{*}}=1 .
$$

We just proved that when $p_{k}=\left(\frac{\bar{U}}{L^{*}}\right)^{\min _{i} \rho_{i}} p_{k}^{*}$ and all other prices are at values specified which are all at most $\bar{U}$, the demand for good $k$ is at least 1 . By Observation 1, no price exceeds $\bar{U}$ during the entire tatonnement process. In complementary markets, since the demand for one good increases when the prices of other goods decrease, we have shown that $x_{k} \geq 1$ if $p_{k} \leq\left(\frac{\bar{U}}{L^{*}}\right)^{\min _{i} \rho_{i}} p_{k}^{*}$.

In the case of the continuous tatonnement, it follows that no price can decrease below the minimum of this value and the initial value of this price. For the discrete case, we argue as follows. Let $\bar{L}_{k}=(1 / 2) \cdot \min \left\{p_{k}^{\circ},\left(\bar{U} / L^{*}\right)^{\min _{i} \rho_{i}} p_{k}^{*}\right\}$. Suppose that Observation 2 were incorrect, then let $t=\tau$ be the first time when some price, say $p_{j}$, is below $\bar{L}_{j}$.

At $t=\tau-1, p_{j}^{\tau-1} \geq \bar{L}_{j}$. But $p_{j}^{\tau-1} \leq 2 \bar{L}_{j}$, as $p_{j}$ can reduce by at most half in one time unit.

Then $x_{j}^{\tau-1} \geq 1$. By the price update rule, $p_{j}^{\tau} \geq p_{j}^{\tau-1} \geq \bar{L}_{j}$, a contradiction.

The lemma now follows from Observation 2.

\section{A.3 Proofs for Substitutes CES}

Proof of Lemma 39. We will use the following bound, which follows from a simple power series expansion: if $c$ is negative and $\left|\Delta p_{j} / p_{j}\right| \leq \min \{1 / 4,1 /|c|\}$,

$$
\left(p_{j}+\Delta p_{j}\right)^{c} \leq p_{j}^{c}+c p_{j}^{c-1}\left(\Delta p_{j}\right)+c(c-1) p_{j}^{c-2}\left(\Delta p_{j}\right)^{2} .
$$

Recall from the proof of Lemma 36 that

$$
D_{\phi}=\phi(p+\Delta p)-\ell_{\phi}(p+\Delta p ; p)=\sum_{j} x_{j} \Delta p_{j}-\sum_{i} \frac{e_{i}}{c_{i}} \log \left(\frac{\sum_{\ell} b_{i \ell}\left(p_{\ell}+\Delta p_{\ell}\right)^{c_{i}}}{S_{i}(p)}\right) .
$$


We apply $(29)$ and the simple bound $\log (1+\epsilon) \leq \epsilon$ for $\epsilon \geq-1$ to yield

$$
\begin{aligned}
D_{\phi} & \leq \sum_{j} x_{j} \Delta p_{j}-\sum_{i} \frac{e_{i}}{c_{i}} \log \left(\frac{\sum_{\ell} b_{i \ell}\left(p_{\ell}^{c_{i}}+c_{i} p_{\ell}^{c_{i}-1} \Delta p_{\ell}+c_{i}\left(c_{i}-1\right) p_{\ell}^{c_{i}-2}\left(\Delta p_{\ell}\right)^{2}\right)}{S_{i}(p)}\right) \\
& =\sum_{j} x_{j} \Delta p_{j}-\sum_{i} \frac{e_{i}}{c_{i}} \log \left(1+c_{i} \sum_{k} \frac{b_{i k}\left(p_{k}\right)^{c_{i}-1}}{S_{i}(p)} \Delta p_{k}+c_{i}\left(c_{i}-1\right) \sum_{k} \frac{b_{i k}\left(p_{k}\right)^{c_{i}-2}}{S_{i}(p)}\left(\Delta p_{k}\right)^{2}\right) \\
& \leq \sum_{j} x_{j} \Delta p_{j}-\sum_{i} \frac{e_{i}}{c_{i}}\left(c_{i} \sum_{k} \frac{x_{i k}}{e_{i}} \Delta p_{k}+c_{i}\left(c_{i}-1\right) \sum_{k} \frac{x_{i k}}{e_{i} p_{k}}\left(\Delta p_{k}\right)^{2}\right) \\
& \leq \sum_{j} x_{j} \Delta p_{j}-\sum_{k} x_{k} \Delta p_{k}+\left(1-c_{\min }\right) \sum_{k} \frac{x_{k}}{p_{k}}\left(\Delta p_{k}\right)^{2} \\
& =\left(1-c_{\min }\right) \sum_{j} \frac{x_{j}}{p_{j}}\left(\Delta p_{j}\right)^{2} .
\end{aligned}
$$

We will need the following bound.

Claim 44. Let $r$ be a fixed number greater than 1 . If $x \leq r-1$, then

$$
\log (1+x) \leq x-\frac{r-1-\log r}{(r-1)^{2}} x^{2} .
$$

Proof. Simply note that $[x-\log (1+x)] / x^{2}$ is a decreasing function for $x \geq 1$.

Proof of Lemma 41. We need the following inequality.

First,

$$
\begin{aligned}
\phi\left(p+\Delta^{*} p\right)-\ell_{\phi}\left(p+\Delta^{*} p ; p\right) & =\sum_{j} x_{j} \Delta^{*} p_{j}-\sum_{i} \frac{e_{i}}{c_{i}} \log \left(\sum_{k} \frac{b_{i k}\left(p_{k}\right)^{c_{i}}}{\sum_{\ell} b_{i \ell}\left(p_{\ell}\right)^{c_{i}}}\left(1+\frac{\Delta^{*} p_{k}}{p_{k}}\right)^{c_{i}}\right) \\
& =\sum_{j} x_{j} \Delta^{*} p_{j}-\sum_{i} \frac{e_{i}}{c_{i}} \log \left(\sum_{k} \frac{e_{i k}}{e_{i}}\left(1+\frac{\Delta^{*} p_{k}}{p_{k}}\right)^{c_{i}}\right) .
\end{aligned}
$$

Note that $\sum_{k} \frac{e_{i k}}{e_{i}}=1$. Thus, by the concavity of the log function, $\log \left(\sum_{k} \frac{e_{i k}}{e_{i}}\left(1+\frac{\Delta^{*} p_{k}}{p_{k}}\right)^{c_{i}}\right) \geq \sum_{k} \frac{e_{i k}}{e_{i}} \log \left(1+\frac{\Delta^{*} p_{k}}{p_{k}}\right)^{c_{i}}$. Then

$$
\begin{aligned}
\phi\left(p^{*}\right)-\ell_{\phi}\left(p^{*} ; p\right) & \geq \sum_{j} x_{j} \Delta^{*} p_{j}-\sum_{i} \frac{e_{i}}{c_{i}} \sum_{k} \frac{e_{i k} c_{i}}{e_{i}} \log \left(1+\frac{\Delta^{*} p_{k}}{p_{k}}\right) \\
& =\sum_{j} x_{j} \Delta^{*} p_{j}-\sum_{k} e_{k} \log \left(1+\frac{\Delta^{*} p_{k}}{p_{k}}\right) \\
& \geq \sum_{j} x_{j} \Delta^{*} p_{j}-\sum_{k} e_{k}\left(\frac{\Delta^{*} p_{k}}{p_{k}}-\frac{r_{k}-1-\log r_{k}}{\left(r_{k}-1\right)^{2}}\left(\frac{\Delta^{*} p_{k}}{p_{k}}\right)^{2}\right) \\
& =\sum_{j} \frac{r_{j}-1-\log r_{j}}{\left(r_{j}-1\right)^{2}} x_{j} \frac{\left(\Delta^{*} p_{j}\right)^{2}}{p_{j}} .
\end{aligned}
$$




\section{B Leontief Lower Bound}

We prove Theorem 26 here.

We consider the following Leontief Fisher market with two buyers and two goods. Buyer 1 has budget $e_{1}=3$ and $b_{11}: b_{12}=1: 3$; buyer 2 has budget $e_{2}=2$ and $b_{21}: b_{22}=2: 1$. There is a unique market equilibrium $\left(p_{1}^{*}, p_{2}^{*}\right)=(0,5)$, with equilibrium demands $\left(x_{11}^{*}, x_{12}^{*}, x_{21}^{*}, x_{22}^{*}\right)=$ $(1 / 5,3 / 5,4 / 5,2 / 5)$. We will show that if tatonnement starts at a carefully chosen price vector, $\left(p_{1}, p_{2}\right)$, the potential function value is $\Theta\left(\left(p_{1}\right)^{2}\right)$ but in the next time step the potential function drops by only $\Theta\left(\left(p_{1}\right)^{3}\right)$.

Let $B=\left\{\left(p_{1}, p_{2} \mid p_{1} \leq \bar{\delta},-\frac{2}{5} p_{1}^{2} \leq p_{1}+p_{2}-5 \leq \frac{2}{5} p_{1}^{2}\right\}\right.$, where $\bar{\delta}>0$ is a sufficiently small positive number which satisfies several conditions stated in the proofs below.

The price update rule of good $j$ is $p_{j}^{t+1}=p_{j}^{t} \cdot e^{z_{j}^{t} / \gamma}$.

Lemma 45. If a tatonnement starts at a price vector in $B$, the set of prices remain in $B$ throughout the whole tatonnement.

Proof. Let $\left(p_{1}, p_{2}\right)$ be a price vector in $B$ and let $\left(p_{1}, p_{2}\right)=\left(\delta, 5-\delta+C \delta^{2}\right)$, where $|C| \leq \frac{2}{5}$. Then the demands are

$$
x_{1}=\frac{3}{15-2 \delta+3 C \delta^{2}}+\frac{4}{5+\delta+C \delta^{2}} \quad x_{2}=\frac{9}{15-2 \delta+3 C \delta^{2}}+\frac{2}{5+\delta+C \delta^{2}} .
$$

Let $\left(p_{1}^{\prime}, p_{2}^{\prime}\right)$ denote the new prices after an update, i.e.

$$
p_{1}^{\prime}=\delta e^{\left(x_{1}-1\right) / \gamma} \quad p_{2}^{\prime}=\left(5-\delta+C \delta^{2}\right) e^{\left(x_{2}-1\right) / \gamma}
$$

The Taylor expansions of $x_{1}, x_{2}, p_{1}^{\prime}, p_{2}^{\prime}$ (with respect to $\delta$ ) are

$$
\begin{gathered}
x_{1}=1-\frac{2}{15} \delta+O\left(\delta^{2}\right), \quad x_{2}=1+\left(\frac{2}{75}-\frac{C}{5}\right) \delta^{2}+O\left(\delta^{3}\right), \\
p_{1}^{\prime}=\delta-\frac{2}{15 \gamma} \delta^{2}+O\left(\delta^{3}\right), \quad p_{2}^{\prime}=5-\delta+\left(C-\frac{C}{\gamma}+\frac{2}{15 \gamma}\right) \delta^{2}+O\left(\delta^{3}\right) .
\end{gathered}
$$

We choose $\bar{\delta}$ to be sufficiently small so that $p_{1}^{\prime}<p_{1}$, and hence $p_{1}^{\prime}<\bar{\delta}$.

The Taylor expansion of $\frac{p_{1}^{\prime}+p_{2}^{\prime}-5}{\left(p_{1}^{\prime}\right)^{2}}$ is

$$
\frac{p_{1}^{\prime}+p_{2}^{\prime}-5}{\left(p_{1}^{\prime}\right)^{2}}=C\left(1-\frac{1}{\gamma}\right)+O(\delta)
$$

We choose $\bar{\delta}$ to be sufficiently small so that

$$
C\left(1-\frac{1}{\gamma}\right)-\frac{1}{10 \gamma} \leq \frac{p_{1}^{\prime}+p_{2}^{\prime}-5}{\left(p_{1}^{\prime}\right)^{2}} \leq C\left(1-\frac{1}{\gamma}\right)+\frac{1}{10 \gamma}
$$

Since $|C| \leq \frac{2}{5}$ and $\gamma \geq 1, C\left(1-\frac{1}{\gamma}\right)-\frac{1}{10 \gamma} \geq-\frac{2}{5}$ and $C\left(1-\frac{1}{\gamma}\right)+\frac{1}{10 \gamma} \leq \frac{2}{5}$. So $\left(p_{1}^{\prime}, p_{2}^{\prime}\right)$ is in $B$.

Lemma 46. If $\left(p_{1}^{t}, p_{2}^{t}\right)$ is in $B$, then $\phi\left(p^{t}\right)-\phi\left(p^{t+1}\right)=\Theta\left(\left(p_{1}\right)^{3}\right)$ and $\phi\left(p^{t}\right)-\phi\left(p^{*}\right)=\Theta\left(\left(p_{1}\right)^{2}\right)$. 
Proof. Let $\left(p_{1}^{t}, p_{2}^{t}\right)=\left(\delta, 5-\delta+C \delta^{2}\right)$. Since the potential function is convex,

$$
\begin{aligned}
\phi\left(p^{t}\right)-\phi\left(p^{t+1}\right) & \leq-\nabla \phi\left(p^{t}\right) \cdot\left(p^{t+1}-p^{t}\right) \\
& =\left(x_{1}-1\right)\left(e^{\left(x_{1}-1\right) / \gamma}-1\right) p_{1}+\left(x_{2}-1\right)\left(e^{\left(x_{2}-1\right) / \gamma}-1\right) p_{2} \\
& =O\left(\frac{p_{1}\left(x_{1}-1\right)^{2}}{\gamma}\right)+O\left(\frac{p_{2}\left(x_{2}-1\right)^{2}}{\gamma}\right) .
\end{aligned}
$$

Recall the Taylor expansions of $x_{1}$ and $x_{2}$. We choose $\bar{\delta}$ to be sufficiently small so that

$$
\left|x_{1}-1\right|=\Theta(\delta), \quad\left|x_{2}-1\right|=O\left(\delta^{2}\right) .
$$

Then

$$
\phi\left(p^{t}\right)-\phi\left(p^{t+1}\right)=\delta \cdot \Theta\left(\frac{\delta^{2}}{\gamma}\right)+\Theta(1) \cdot O\left(\frac{\delta^{4}}{\gamma}\right)=\frac{1}{\gamma} \Theta\left(\delta^{3}\right)
$$

Next, we will show that the potential function is $\Theta\left(\delta^{2}\right)$. The following derivation is similar to the one for the upper sandwiching bound. Let $\Delta^{*} p_{\ell}=p_{\ell}^{*}-p_{\ell}$. Recall that

$$
\phi\left(p^{*}\right)-\ell_{\phi}\left(p^{*} ; p\right)=\sum_{j} x_{j} \Delta^{*} p_{j}-\sum_{i} e_{i} \log \left(1+\frac{\sum_{\ell} b_{i \ell} \Delta^{*} p_{\ell}}{\sum_{\ell} b_{i \ell} p_{\ell}}\right) .
$$

We choose $\bar{\delta}$ to be sufficiently small so that $\frac{3}{4} \leq \frac{\sum_{\ell} b_{i \ell}\left(p_{\ell}+\Delta^{*} p_{\ell}\right)}{\sum_{\ell} b_{i \ell} p_{\ell}} \leq \frac{5}{4}$. Then we can use (24) to obtain

$$
\begin{aligned}
\phi\left(p^{*}\right)-\ell_{\phi}\left(p^{*} ; p\right) & \leq \sum_{j} x_{j} \Delta^{*} p_{j}-\sum_{i} e_{i}\left[\frac{\sum_{\ell} b_{i \ell} \Delta^{*} p_{\ell}}{\sum_{\ell} b_{i \ell} p_{\ell}}-\frac{2}{3}\left(\frac{\sum_{\ell} b_{i \ell} \Delta^{*} p_{\ell}}{\sum_{\ell} b_{i \ell} p_{\ell}}\right)^{2}\right] \\
& =\frac{2}{3} \sum_{i} e_{i}\left(\sum_{\ell} \frac{x_{i \ell}}{e_{i}} \Delta^{*} p_{\ell}\right)^{2}=\frac{2}{3} \sum_{i} \frac{1}{e_{i}}\left(\sum_{\ell} x_{i \ell} \Delta^{*} p_{\ell}\right)^{2} .
\end{aligned}
$$

Note that $\Delta^{*} p_{1}=-\delta$ and $\Delta^{*} p_{2}=\delta-C \delta^{2}$. The taylor expansions for the $\left\{x_{i j}\right\}$ are

$$
x_{11}=\frac{1}{5}+O(\delta) \quad x_{12}=\frac{3}{5}+O(\delta) \quad x_{21}=\frac{4}{5}+O(\delta) \quad x_{22}=\frac{2}{5}+O(\delta) .
$$

Hence, the Taylor expansions of $\frac{1}{e_{i}}\left(\sum_{\ell} x_{i \ell} \Delta^{*} p_{\ell}\right)^{2}$ are

$$
\frac{1}{e_{1}}\left(x_{11} \Delta^{*} p_{1}+x_{12} \Delta^{*} p_{2}\right)^{2}=\frac{4}{75} \delta^{2}+O\left(\delta^{3}\right), \quad \frac{1}{e_{2}}\left(x_{21} \Delta^{*} p_{1}+x_{22} \Delta^{*} p_{2}\right)^{2}=\frac{2}{25} \delta^{2}+O\left(\delta^{3}\right) .
$$

Thus

$$
\phi\left(p^{*}\right)-\ell_{\phi}\left(p^{*} ; p\right) \leq \frac{4}{45} \delta^{2}+O\left(\delta^{3}\right)
$$

Then

$$
\begin{aligned}
\phi\left(p^{*}\right)-\phi(p) & \leq \frac{4}{45} \delta^{2}+O\left(\delta^{3}\right)-z_{1} \Delta^{*} p_{1}-z_{2} \Delta^{*} p_{2} \\
& =\frac{4}{45} \delta^{2}-\left(-\frac{2}{15} \delta\right)(-\delta)-\left(\frac{2}{75}-\frac{C}{5}\right) \delta^{2} \cdot\left(\delta-C \delta^{2}\right)+O\left(\delta^{3}\right) \\
& =-\frac{2}{45} \delta^{2}+O\left(\delta^{3}\right) .
\end{aligned}
$$

We can choose $\bar{\delta}$ sufficiently small so that $\phi(p)-\phi\left(p^{*}\right)=\Theta\left(\delta^{2}\right)$. 
Proof. (of Theorem 26,) By Lemma 46, it takes $\Theta\left(1 / p_{1}\right)$ steps for $\phi(p)$ to halve. So starting at $p_{1}=\bar{\delta}$, to reduce $\phi(p)$ by a $2^{i}$ factor takes $\Theta\left(\left[1+\sqrt{2}+\ldots+\sqrt{2^{i}}\right] \cdot[1 / \sqrt{\bar{\delta}}]\right)=\Theta\left(\sqrt{2^{i} / \bar{\delta}}\right)$ steps. In other words,

$$
\phi\left(p^{t}\right)-\phi\left(p^{*}\right)=\Theta\left(\frac{\phi\left(p^{0}\right)-\phi\left(p^{*}\right)}{t^{2} \bar{\delta}}\right)=\Theta\left(\frac{\phi\left(p^{0}\right)-\phi\left(p^{*}\right)}{t^{2}}\right) .
$$

